\title{
RANS Analyses of Turbofan Nozzles with Wedge Deflectors for Noise Reduction
}

\author{
James R. DeBonis * \\ NASA Glenn Research Center, Cleveland, Ohio 44135
}

\begin{abstract}
Computational fluid dynamics (CFD) was used to evaluate a promising concept for reducing the noise at take-off of dual-stream, turbofan nozzles. The concept, offset stream technology, reduces the jet noise observed on the ground by diverting (offsetting) the majority of the fan flow below the core flow, thickening this layer between the high velocity core flow and the ground observers. In this study a wedge placed in the internal fan stream is used as the diverter. Wind, a Reynolds Averaged Navier-Stokes (RANS) code, was used to analyze the flowfield of the exhaust plume and to calculate nozzle performance. Results showed that the wedge effectively diverts the fan flow and the turbulent kinetic energy on the observer side of the nozzle is reduced. The reduction in turbulent kinetic energy should correspond to a reduction in noise. The blockage due to the wedge reduces the fan massflow proportional to its blockage and the overall thrust is consequently reduced. The CFD predictions are in very good agreement with experimental data. This noise reduction concept shows promise for reduced jet noise at a small reduction in thrust. It has been demonstrated that RANS CFD can be used to optimize this concept.
\end{abstract}

\section{Nomenclature}

$\begin{array}{ll}k & \text { turbulent kinetic energy } \\ \dot{m} & \text { massflow } \\ p & \text { pressure } \\ x, y, z & \text { cartesian coordinates } \\ B P R & \text { bypass ratio } \\ C_{d} & \text { discharge coefficient } \\ C_{f g} & \text { thrust coefficient } \\ D & \text { diameter } \\ \mathrm{F} & \text { nozzle force (thrust) } \\ M & \text { Mach number } \\ N P R & \text { nozzle pressure ratio } \\ T & \text { temperature } \\ U & \text { ideal exit velocity } \\ & \\ \text { subscripts } & \\ \text { actual } & \text { measured quantity } \\ \text { baseline } & \text { nozzle without flow deflectors } \\ \text { ideal } & \text { isentropically expanded to freestream condition } \\ c & \text { core } \\ f & \text { fan } \\ 0 & \text { stagnation/total condition } \\ \infty & \text { freestream condition }\end{array}$

\footnotetext{
*Aerospace Engineer, Inlet \& Nozzle Branch, 21000 Brookpark Rd., MS 5-12, Associate Fellow AIAA
} 


\section{Introduction}

The concept of offsetting the fan stream of a dual flow turbofan nozzle to reduce jet noise was developed by Papamoschou at the University of California Irvine (UCI).$^{1-3}$ The idea is to provide a thicker layer of fan flow on the lower (observer) side of the nozzle. This thick layer of low speed flow reduces the convective Mach number of the turbulent eddies generated by the high speed core flow. This reduces these turbulent structures' ability to generate strong acoustic waves that travel to the far field (on the lower side of the nozzle). His initial concept was to physically offset the nozzle flow paths. This eccentric nozzle would be difficult to implement in a real system. He found that vanes and/or wedges placed in the fan stream can deflect the fan flow to the lower portion of the jet to achieve the desired flow field and resulting acoustics in a simple easy to implement manner.

Papamoschou tested these concepts in a small jet facility (1 inch fan exit diameter) and measured jet noise reductions of over $5.0 \mathrm{~dB}$ in overall sound pressure level. These impressive results led the National Aeronautics and Space Administration (NASA) to examine this noise reduction concept at a larger scale. NASA developed the Offset Stream Technology (OST) program to design and test turbofan nozzles with devices to offset the fan flow. The program consisted of computational fluid dynamics (CFD) analysis to screen flow deflector design and placement and to estimate nozzle performance, design of experiments (DOE) methodology to reduce the number of experimental test points required, additional small scale testing, and large scale testing (9.63 inch fan exit diameter) in the NASA Glenn Nozzle Acoustic Test Rig (NATR).

There are four other papers from the OST study that form a complete description of the program. A companion CFD study examining the vane deflector and s-duct nozzles was performed by Dippold et al. ${ }^{5}$ The experimental results from NATR, acoustics and Particle Image Velocimetry (PIV) based velocity measurements in the exhaust plume, were reported by Brown et $\mathrm{al}^{6}$ and a comparison of experimental results between UCI and NASA Glenn was detailed by Zaman et al. ${ }^{7}$ The results of the DOE study were reported by Henderson et al. ${ }^{8}$

The experimental results at NATR indicate that the wedge deflectors do reduce the noise on the lower side of the nozzle at downstream angles. However, at other angles on the lower side and all angles on the sideline and upper side the noise was increased. In general, the overall estimated perceived noise level (EPNL) increased relative to the baseline due to the higher noise at the other locations. One configuration, $B P R=5.0, M_{\infty}=0.0$, showed a small noise reduction of $0.8 \mathrm{EPN} \mathrm{dB}$. Based on measurements of turbulent kinetic energy, they concluded that the wedge designs tested were too aggressive.

Thrust performance is a critical factor in assessing low noise nozzle concepts. Papamoschou estimated the thrust loss in nozzles with vane deflectors based on airfoil drag and nozzle flow deflection ${ }^{2}$ and he and co-workers experimentally examined the aerodynamics of a wedge deflector in a simplified configuration. ${ }^{9}$ But up to this point there has been no detailed examination of the performance of these nozzles. The NATR tests did not include force measurements. A key element of the CFD calculations presented here was to characterize the thrust of these nozzles and compare them to a baseline nozzle without deflectors. This work represents the highest fidelity estimates of Offset Stream nozzle performance to date.

This paper describes the CFD analyses performed to support the design and testing of turbofan nozzles with the wedge shaped flow deflectors. The CFD analyses consisted of 3 parts: (1) pre-test predictions to verify the wedge design and its effect on the flow field (2) performance calculations to examine the performance penalties due to the noise reduction scheme (3) validation of the CFD methodology against experimental data.

\section{Description of Nozzles and Flow Conditions}

The nozzles used were originally designed at the Glenn Research Center for an experimental study of jet noise reduction using chevrons. ${ }^{10}$ The baseline nozzles with no chevrons were used. For this study a wedge flow deflector was placed in the fan stream. The wedge sits completely inside the nozzle and the base of the wedge is coincident with the nozzle exit (figure 1). Three different nozzles were analyzed: 3BB, 4BB and 5BB (figure 2). The 3BB and 5BB nozzles both have an external nozzle plug in the core stream and have nozzle bypass ratios $(B P R)$ of five and eight respectively. These two nozzles share the same fan flow geometry. The core flow path was altered to affect the bypass ratio change. The $4 \mathrm{BB}$ nozzle has an internal plug in the core flow and has a bypass ratio of eight. This nozzle was studied computationally, but not tested in the experiment. All three nozzles have the same fan diameter of 9.63 inches. 
The shape of the wedge deflector studied here is the same as the wedge used by Papamoschou in his initial studies. The wedge has a half angle of $11^{\circ}$ and a base width of 3.44 inches. The upper and lower surfaces of the wedge conform to the internal contours of the fan stream. The wedge reduces the exit area of the fan stream by 14 percent. Two additional wedges were tested in the experiment, but not analyzed. These wedges both had half angles of $20^{\circ}$ and base widths of 3.44 inches and 2.23 inches.

The calculations performed for the pre-test predictions and performance analyses were done using cycle data for a representative takeoff condition. Details of the operating conditions are in table 1 . The wedge deflector would most likely be implemented by a system of flaps installed in the nozzle pylon. The deflector would be removed from the fan stream at cruise and the nozzle would operate normally. Thus, no predictions of performance were necessary at cruise conditions. The experimental data were taken at slightly different conditions and the analyses were rerun at these conditions for code validation purposes (table 2). For the 3BB validation case the nozzle was tested with static freestream conditions. This analysis was run with a freestream Mach number of 0.05 for numerical stability. Two 5BB cases were run, one with heated streams and one with cold streams. Both cases had a freestream Mach number of 0.20.

\section{Analysis Procedure}

\section{A. Geometry Definition}

The computational grids (figure 3 ) were generated using Gridgen version 15 software. ${ }^{11}$ The database, which provides the geometry for the grid generator, was taken directly from the computer aided drafting (CAD) file used to design and build the experimental model hardware. The grids are a structured multi-block type with separate grid blocks representing the following: (1) internal primary flowpath, (2) the external primary flow path including the plug, (3) the internal secondary flow path, (4) the external secondary flow path, (5) the nacelle, and (6) the jet plume region (figure 4). Dimension of the grids are given in table 3. At block interfaces the grids are not contiguous and a Roe-based zone coupling algorithm is used to pass the fluxes between blocks.

The grids for the baseline nozzles with no wedge deflector were planar, taking advantage of the axisymmetric geometry. A total of 66,734 points were used. The grids for the nozzles with the wedge deflector were three-dimensional: a 180 degree segment, taking advantage of the symmetry about the xy-plane (vertical streamwise) plane. A total of 6,072,794 grid points were used.

\section{B. Flow Solution}

The flow solver used for all the computations was Wind version 5.0. ${ }^{12}$ Wind is a general purpose Reynolds Averaged Navier-Stokes equation solver. All the calculations presented here used the same code options. The default numerical scheme was used: implicit time-stepping and a second-order physical Roe scheme for stretched grids. Turbulence was modeled using the Menter Shear Stress Transport (SST) model. ${ }^{13}$ No additional corrections (ie. compressibility corrections) were used in the model.

Grid sequencing in all three computational directions was used during the flow solution. Sequencing solves the equations on a reduced grid to speed convergence. The sequenced results are then interpolated onto the finer grid for continued processing. One level of sequencing involves removing every other grid point from the previous level. The solution was started at two levels of sequencing and progressed to the full grid solution. Nozzle force measurements were monitored and the sequencing was reduced when the nozzle force converged on that grid level. Nozzle force measurements between the final grid level and the next sequenced level agreed within one percent, indicating that grid convergence was obtained. Local time stepping was used to achieve a steady state solution. For the 3BB case at near static ambient conditions $\left(M_{\infty}=0.05\right)$

the solution did not converge in this manner. For this case the solution was switched to global time stepping and convergence to a steady state was achieved.

\section{Results}

\section{A. Examination of the Flowfields}

Contours of axial velocity and turbulent kinetic energy in the streamwise (xy-plane) and cross-planes (yzplanes) downstream of the nozzle exit are presented to illustrate the effect of the wedge deflector on the flow 
field (figures 5 - 16). Data is presented for all three nozzles (3BB, 4BB and 5BB) and the results show that all three flowfields behave similarly.

Velocity contours in the streamwise planes compare the wedge nozzle to the baseline nozzle (figures $5-7$ ). These contours show that the presence of the wedge directs the core flow upward and significantly shortens the jet's potential core. The layer of fan flow on the underside of the jet plume is substantially thicker. A low-velocity region immediately behind the wedge indicates a large recirculation region is present there.

Turbulent kinetic energy contours in the streamwise planes are shown in figures $8-10$. On the upper side of the exhaust plume levels of $k / U_{c}^{2}$ are increased and the peak level has moved upstream, closer to the nozzle exit. On the lower side of the exhaust plume the levels of $k / U_{c}^{2}$ have been reduced. This is the desired effect. A small local peak in turbulence levels is seen directly behind the wedge, again indicating a separated region and potential noise source.

The movement of the fan flow induced by the wedge deflector is more easily seen in cross-planes downstream of the nozzle exit (figures 11 - 16). The fan flow moves to the lower half of the exhaust plume and at $x / D_{f}=5.0$ the fan stream is almost completely below the core flow. The upper portion of the core flow comes in direct contact with the freestream flow and is not buffered by the fan flow. This results in high turbulence levels and will certainly increase the noise on this side of the nozzle. At $x / D_{f}=3.0$ the high turbulence levels are not shielded by the fan flow from the top of the nozzle down to the sideline position $\left(0^{\circ}\right.$ to $90^{\circ}$, measured from the vertical). The turbulent kinetic energy levels resulting from the core stream interacting directly with the freestream are on the order of an isolated jet operating at the core stream conditions and could result in very high noise levels at sideline and above the nozzle. The lower side has much lower turbulence levels and should result in lower noise on this side of the exhaust plume. The CFD results agree with the experimentalists' conclusion that the current wedge design is too aggressive. ${ }^{6}$ It appears that the wedge moved too much fan flow, exposing the core flow to the freestream. Lower wedge angles should be investigated to reduce the deflection of the fan flow. Also, shorter wedges that do not span the entire fan duct height may prevent the core flow from being completely exposed to the freestream.

\section{B. Nozzle Performance}

\section{Calculation Procedure}

Nozzle performance was computed using the Wind utility, CFPOST. Stream thrust was computed at the fan and core exits. Pressure and viscous forces were computed on the remaining surfaces: core nozzle lip, fan nozzle lip, plug, splitter between fan and core, nacelle, and wedge base.

Nozzle performance will be reported in two ways. First, the total thrust of the nozzle with wedge deflector will be expressed as a fraction of the baseline nozzle thrust

$$
F / F_{\text {baseline }}
$$

Second, it will be expressed in terms of a thrust coefficient. Typically nozzle performance results are reported using the gross thrust coefficient.

$$
C_{f g}=\frac{F_{\text {actual }}}{\dot{m}_{\text {actual }} U_{\text {ideal }}}
$$

Using the actual massflow in the denominator, $C_{f g}$, removes the deviation from the ideal massflow from the performance measure. Discharge coefficient is a separate measure for the reduction in massflow.

$$
C_{d}=\frac{\dot{m}_{\text {actual }}}{\dot{m}_{\text {ideal }}}
$$

For subsonic nozzles the massflow through the nozzle can vary due to downstream effects and the thrust coefficient does not give a true measure of nozzle performance. In these circumstances, the change in the measured thrust is caused by the change in the nozzle flow rate and not a change in exit velocity. This is especially true for two-stream nozzles where the over- or under-expansion of one stream can affect the flow rate of the other. Nozzles with noise suppressing features can also affect flow rates. Therefore, a thrust coefficient that accounts for the change in massflow rate is used in this paper. It is simply a thrust coefficient based on the ideal massflow rather than the actual massflow. It can be conveniently written as the product of the gross thrust coeffcient and discharge coefficient.

$$
\begin{gathered}
\frac{F_{\text {actual }}}{\dot{m}_{\text {ideal }} U_{\text {ideal }}}=\frac{\dot{m}_{\text {actual }}}{\dot{m}_{\text {ideal }}} \frac{F_{\text {actual }}}{\dot{m}_{\text {actual }} U_{\text {ideal }}}=C_{d} C_{f g} \\
\text { American Institute of Aeronautics and Astronautics }
\end{gathered}
$$


A baseline case, no wedge, was calculated for each nozzle geometry. Performance of the wedge nozzles is referenced to the baseline and is reported as $\Delta\left(C_{d} C_{f g}\right)$

$$
\Delta\left(C_{d} C_{f g}\right)=\left(C_{d} C_{f g}\right)_{\text {wedge }}-\left(C_{d} C_{f g}\right)_{\text {baseline }}
$$

\section{Performance Results}

Thrust of the wedge nozzles expressed as a ratio to the baseline nozzle thrust is shown in figure 17(a). The wedge has created thrust losses between 11 and 13 percent. The reason for these losses is clear. Figure 17(b) shows the loss in massflow of wedge nozzles, expressed as a ratio to the baseline nozzles. Total massflow reduction also varies between 11 and 13 percent. This reduction is due entirely to the reduction in fan massflow, which is approximately $14 \%$ for all three cases. These losses are a direct result of the blockage created by the wedge ( $14 \%$ of the fan exit area). For this noise reduction concept to be viable, the engine would have to be oversized to accommodate the performance penalty at takeoff. Reducing the size of the wedge, as suggested for acoustic reasons, would reduce the thrust penalty. An alternative concept, placing the wedge external to the fan nozzle on the splitter, has been proposed and should eliminate this loss of fan massflow. ${ }^{14}$

Nozzle performance expressed as a thrust coefficient, $C_{d} C_{f g}$, for both the baseline and wedge configurations for all three nozzles is shown in figure 18(a). For the wedge nozzles, this performance measure uses the ideal conditions based on the actual exit area and therefore does not include the loss due to blockage. The external plug nozzles have uniformly higher performance than the internal plug nozzle. Figure 18(b) shows the decrement in performance due to the wedge. The losses due to the wedge vary from about 0.7 to 1.1 percent and appear to be relatively low considering the large base area created by the wedge. A detailed breakout of the forces that comprise the thrust calculation are shown for the 5BB baseline and wedge nozzles in figure 19. The figure confirms that the primary loss mechanism is the loss in fan stream thrust due to the reduced massflow. The presence of the wedge reduces the positive force on the nozzle plug and increases the drag on the nacelle. The base drag on the wedge surface is a relatively minor component. This is somewhat surprising but can be explained by the streamlines in figure 20. The figure shows that the flow which separates and recirculates behind the base of the wedge is the external flow over the nacelle. This also shows the linkage between the presence of the wedge and the increased nacelle drag (figure 19). The fan flow, the primary contributor to the thrust, is turned by the wedge to create the offset stream but does not separate behind the wedge, which would result in large thrust losses.

By examining nozzle performance using the $C_{d} C_{f g}$ parameter we can see that the losses created by the wedge deflector would be acceptable if the reduction in massflow could be removed or accounted for in the nozzle design. The wedge deflector could be a viable noise reduction concept if the wedge size could be significantly reduced or an external wedge that did not reduce the fan flow rate was employed.

The wedge induces a downward movement of the fan flow and a corresponding upward movement of the core flow. The resulting vertical component of the nozzle thrust is presented in figure 21 . The data indicates that the wedge induces a very small vertical component to the thrust. The external plug nozzles have a smaller component, about $0.5 \%$ of the axial thrust, and the internal plug has a slightly larger component, approximately $1.5 \%$ of the axial thrust.

\section{Comparison to Experiment}

Once the experimental test program was carried out the CFD analyses were rerun to match the experimental conditions. The same computational grids, code version, and code options were used. The experimental data were withheld until the predictions were complete and no attempts were made to adjust code parameters to better match the data.

The experimental data consisted of particle image velocimetry measurements of instantaneous velocity components in the nozzle plume. ${ }^{15}$ The data were measured in planes parallel to the nozzle exit at distances of 1.0, 1.5, 2.0, 3.0, 5.0 and 7.0 fan diameters downstream of the fan nozzle exit. Contours of u-velocity (axial component) and turbulent kinetic energy are compared for both the baseline and wedge configurations for the following nozzles and flow conditions: 3BB nozzles at hot flow conditions and a static freestream (figures 22 - 25), 5BB nozzles at hot flow conditions and a Mach 0.20 freestream (figures 26 - 29), and 5BB nozzles at cold flow conditions and a Mach 0.20 freestream (figures $30-33$ ).

In general, very good agreement is achieved between the CFD and experiment in terms of plume shape, velocity levels and turbulent kinetic energy levels. In particular, the predictions for the baseline nozzles 
match the experiment very closely. For the wedge nozzles, velocity levels and plume shape also are predicted well. The CFD does over-predict the levels of turbulent kinetic energy for the 3BB wedge case and underpredicts the levels for both $5 \mathrm{BB}$ wedge cases (hot and cold) in the upper portion of the jet where the core flow interacts directly with the freestream. One would expect the levels of $k / U_{c}^{2}$ to decrease with increasing freestream Mach number. It is unclear why the experiment showed the opposite trend. The good agreement between the analysis and the experiment is very encouraging and indicates that this RANS CFD technique can be used effectively to optimize the wedge nozzle design and accurately predict the resulting flowfields.

\section{Summary and Conclusions}

Detailed Reynolds Averaged Navier-Stokes calculations of turbofan nozzles with wedge deflectors for noise reduction were carried out to support NASA's Offset Stream Technology program. The nozzles were designed to reduce jet noise at takeoff conditions. The computational fluid dynamics calculations were intended to characterize the exhaust plume aiding in wedge design, predict nozzle thrust performance and validate the computational technique against experimental data.

The predicted flowfields showed that the wedge deflector was effective at redirecting the fan flow to the underside of the exhaust plume, effectively reducing noise on this side of the nozzle. The predictions indicate that the wedge design is too aggressive, deflecting too much flow and exposing the core flow to the freestream on the top and sides of the plume. Predictions of turbulent kinetic energy suggest that noise levels at the sideline and upper side of the nozzle may approach those of an isolated jet at core flow conditions.

Nozzle performance calculations show that the thrust of the nozzle is significantly reduced relative to an unmodified nozzle. This thrust loss is caused by a reduction in massflow of the fan stream which is directly proportional to the area reduction of the fan exit caused by the wedge. Thrust losses imparted by the wedge not due to the fan flow reduction are relatively small. If the loss in massflow could be removed or accounted for in the nozzle design the wedge concept would be viable in terms of performance.

Predictions of axial velocity and turbulent kinetic energy in several downstream planes parallel to the nozzle exit were compared to experimentally obtained data. Agreement for both quantities was very good for all nozzle configurations, locations and flow conditions considered.

Acoustic testing of the nozzles considered here showed that they did not achieve the desired noise reduction. Based on the predicted flowfields, performance calculations and agreement with experimental data, this study clearly shows that the CFD methodology used here can be successfully used to help redesign the nozzle to achieve the desired flowfield, nozzle performance and noise reduction.

\section{Appendix: Performance of all Offset Stream Technology Nozzles}

The wedge deflectors were one of three devices studied in the OST program to offset the fan flow. The other methods used were vane deflectors and an offset fan duct (s-duct). A separate paper details the parallel CFD analyses including performance predictions for these two concepts. ${ }^{5}$ Computations for the vane deflectors included a detailed optimization. The performance predictions of all the OST nozzle concepts are compared here.

The analysis code, numerical scheme, turbulence model and post processing techniques used in both studies were identical. But the computational grids used for the vane and s-duct case differed from those in the wedge study. There were two primary differences that resulted in a difference in predictions of nozzle thrust. First, the wedge grids resolved the trailing edge of the nozzle lip whereas the other grids treated them as sharp edges. Second, the present grids located the farfield boundary 10 fan diameters away from the nozzle, much further than the other grids. To account for these differences, each OST nozzle calculation was referenced to a baseline nozzle calculation using the same grid topology. When the nozzle performance is referenced to the equivalent baseline performance, the grid differences are removed.

Figure 34 compares the performance of all OST noise reduction concepts. When compared on a thrust ratio basis, the wedge nozzles are clearly the worst performers due to the loss in massflow discussed earlier. The vane deflectors performed the best. This is not surprising, due to the fact that the vane design and placement were subject to a detailed optimization studied using design of experiments. Vane A, the initial design, has the poorest performance and the difference between it and Vane C, the best performer, shows the improvements possible through a CFD-based optimization. When the blockage effect of the wedge is not considered, the thrust coefficient indicates that the losses for the OST nozzle concepts are similar. 
Optimization of the wedge or s-duct designs have the potential to increase their performance, comparable to the vane deflectors.

\section{References}

${ }^{1}$ Papamoschou, D., "Noise Suppression in Moderate-Speed Multistream Jets," AIAA Paper 2002-2557, 2002.

${ }^{2}$ Papamoschou, D., "New Method for Jet Noise Reduction in Turbofan Engines," AIAA Journal, Vol. 42, No. 11, 2004, pp. 2245-2253.

${ }^{3}$ Papamoschou, D. and Nishi, K., "Jet Noise Suppression with Fan Flow Deflectors in Realistic-Shaped Nozzle," AIAA Paper 2005-993, 2005.

${ }^{4}$ Castner, R., "The Nozzle Acoustic Test Rig; An Acoustic and Aerodynamic Free-Jet Facility," NASA TM $106495,1994$.

${ }^{5}$ Dippold, V., Foster, L., and Wiese, M., "Computational Analyses of Offset Stream Nozzles for Noise Reduction," AIAA Paper 2007-3589, 2007.

${ }^{6}$ Brown, C., Bridges, J., and Henderson, B., "Offset Stream Technology Test - Summary of Results," AIAA Paper 2007$3664,2007$.

${ }^{7}$ Zaman, K., Bridges, J., and Papamoschou, D., "Offset Stream Technology - Comparison of Results from UC and GRC Experiments," AIAA Paper 2007-438, 2007.

${ }^{8}$ Henderson, B., Norum, T., and Bridges, J., "An MDOE Assessment of Nozzle Vanes for High Bypass Ratio Jet Noise Reduction," AIAA Paper 2006-2543, 2006.

${ }^{9}$ Papamoschou, D., Vu, A., and Johnson, A., "Aerodynamics of Wedge-Shaped Deflectors for Jet Noise Reduction," AIAA Paper 2006-3655, 2006.

${ }^{10}$ Saiyed, N., "Separate Flow Nozzle Test Status Meeting," NASA CP 2000-210524, 2000.

${ }^{11}$ Pointwise, Inc., http://www.pointwise.com/gridgen/.

${ }^{12}$ The NPARC Alliance, http://www.grc.nasa.gov/www/winddocs/.

${ }^{13}$ Menter, F. R., "Two-Equation Eddy-Viscosity Turbulence Models for Engineering Applications," AIAA Journal, Vol. 32, No. 8, 1994, pp. 1598-1605.

${ }^{14}$ Papamoschou, D., "Fan Flow Deflection in Simulated Turbofan Exhaust," AIAA Journal, Vol. 44, No. 12, 2006, pp. 30883097.

${ }^{15}$ Wernet, M. and Bridges, J., "Application of DPIV to Enhanced Mixing Heated Nozzle Flows," AIAA Paper 2002-0691, 2002. 


\begin{tabular}{lc|ccc|ccc|ccc} 
& & \multicolumn{3}{|c|}{ Fan } & \multicolumn{3}{c|}{ Core } & \multicolumn{3}{c}{ Freestream } \\
\hline Nozzle & $B P R$ & $N P R$ & $T_{0}(\mathrm{R})$ & $U(f t / s)$ & $N P R$ & $T_{0}(\mathrm{R})$ & $U(f t / s)$ & $p_{\infty}\left(l b / f t^{2}\right)$ & $T_{\infty}(\mathrm{R})$ & $M_{\infty}$ \\
\hline 3BB & 5.0 & 1.83 & 655 & 1117 & 1.68 & 1500 & 1575 & 2059 & 530 & 0.29 \\
4BB & 8.0 & 1.57 & 625 & 953 & 1.52 & 1520 & 1435 & 2059 & 530 & 0.29 \\
5BB & 8.0 & 1.62 & 640 & 995 & 1.42 & 1498 & 1310 & 2059 & 530 & 0.29
\end{tabular}

Table 1. Nozzle operating conditions for performance calculations

\begin{tabular}{lc|ccc|ccc|ccc} 
& & \multicolumn{3}{|c|}{ Fan } & \multicolumn{3}{c|}{ Core } & \multicolumn{3}{c}{ Freestream } \\
\hline Nozzle & $B P R$ & $N P R$ & $T_{0}(\mathrm{R})$ & $U(f t / s)$ & $N P R$ & $T_{0}(\mathrm{R})$ & $U(f t / s)$ & $p_{\infty}\left(l b / f t^{2}\right)$ & $T_{\infty}(\mathrm{R})$ & $M_{\infty}$ \\
\hline 3BB hot & 5.0 & 1.83 & 640 & 1104 & 1.68 & 1500 & 1575 & 2059 & 537 & 0.05 \\
5BB hot & 8.0 & 1.62 & 640 & 995 & 1.42 & 1498 & 1310 & 2059 & 537 & 0.20 \\
5BB cold & 8.0 & 1.42 & 536 & 783 & 1.86 & 536 & 1021 & 2059 & 537 & 0.20
\end{tabular}

Table 2. Nozzle operating conditions for comparison to experiment

\begin{tabular}{r|r|r|r|r} 
Block & Axial & Radial & Azimuthal & Total \\
\hline 1 & 73 & 45 & 91 & 298,935 \\
2 & 53 & 165 & 91 & 795,795 \\
3 & 65 & 45 & 91 & 266,175 \\
4 & 45 & 109 & 91 & 446,355 \\
5 & 69 & 45 & 91 & 282,555 \\
6 & 253 & 173 & 91 & $3,982,979$ \\
\hline Wedge (3D) & & & & $6,072,794$ \\
\hline Baseline & & & & 66,734
\end{tabular}

Table 3. Dimensions of the computational grid 


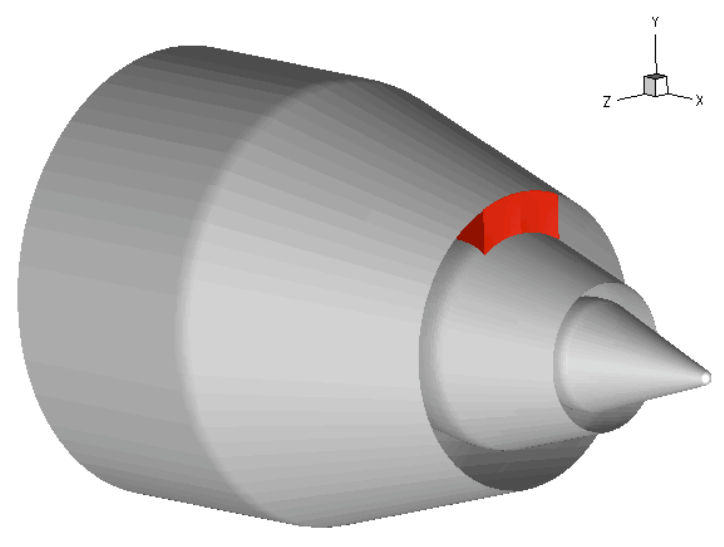

Figure 1. Turbofan nozzle with wedge installed in the fan stream

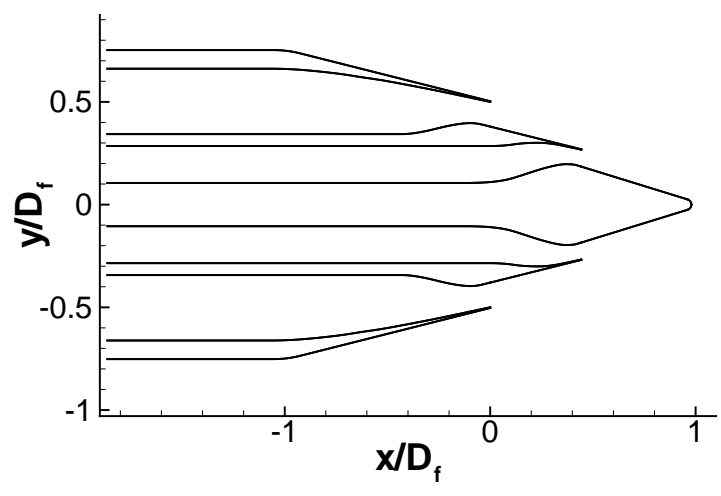

(a) 3BB: external plug, $B P R=5.0$

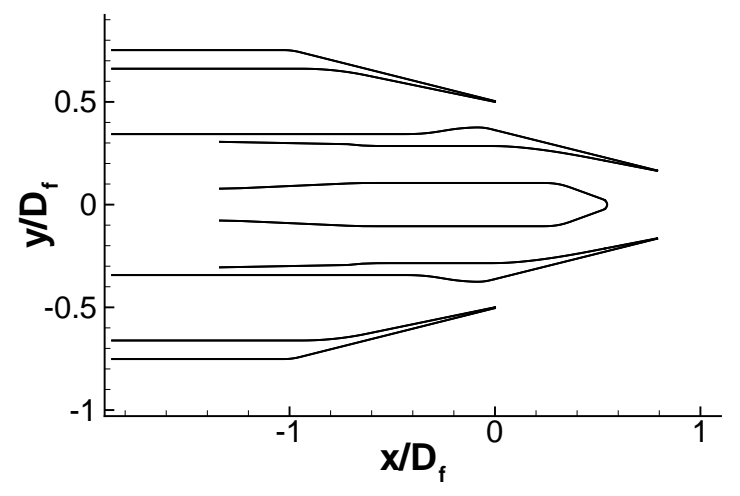

(b) $4 \mathrm{BB}$ : internal plug, $B P R=8.0$

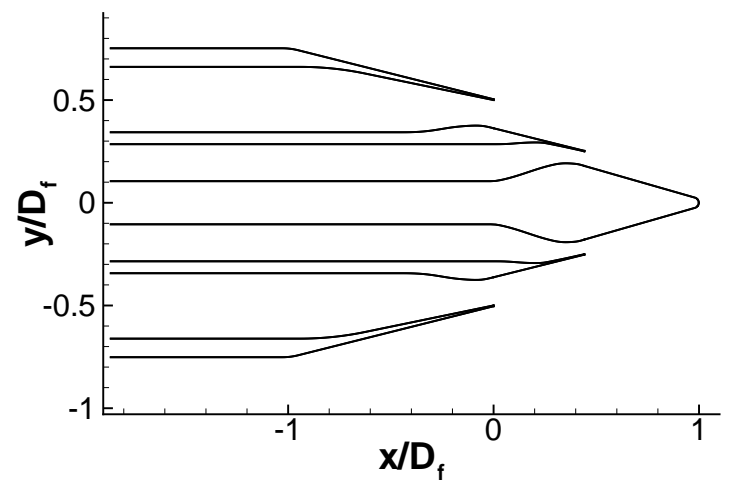

(c) 5BB: external plug, $B P R=8.0$

Figure 2. Schematic drawings of NASA GRC turbofan nozzles 


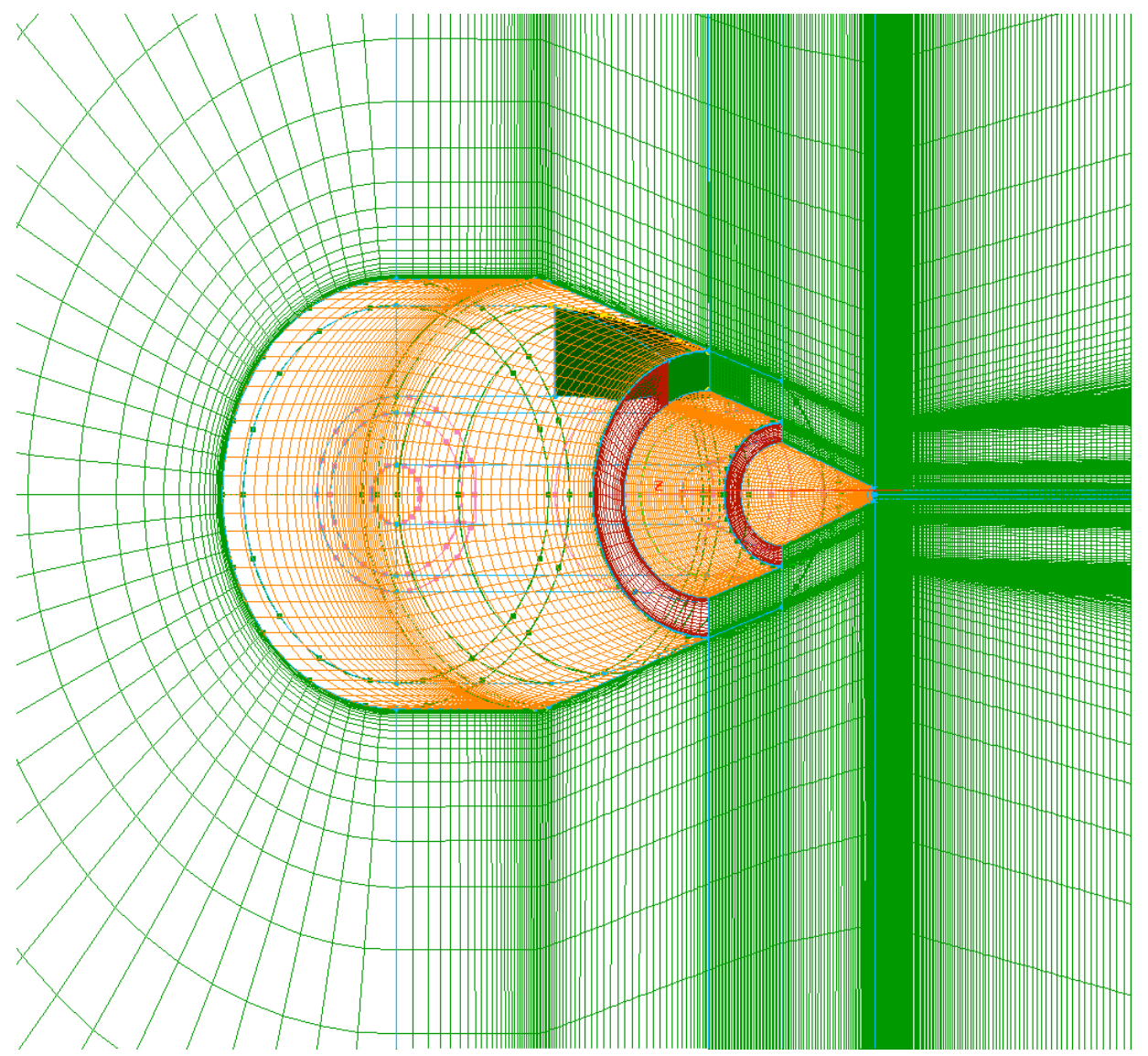

Figure 3. Grid of 5BB nozzle with wedge installed in the fan stream

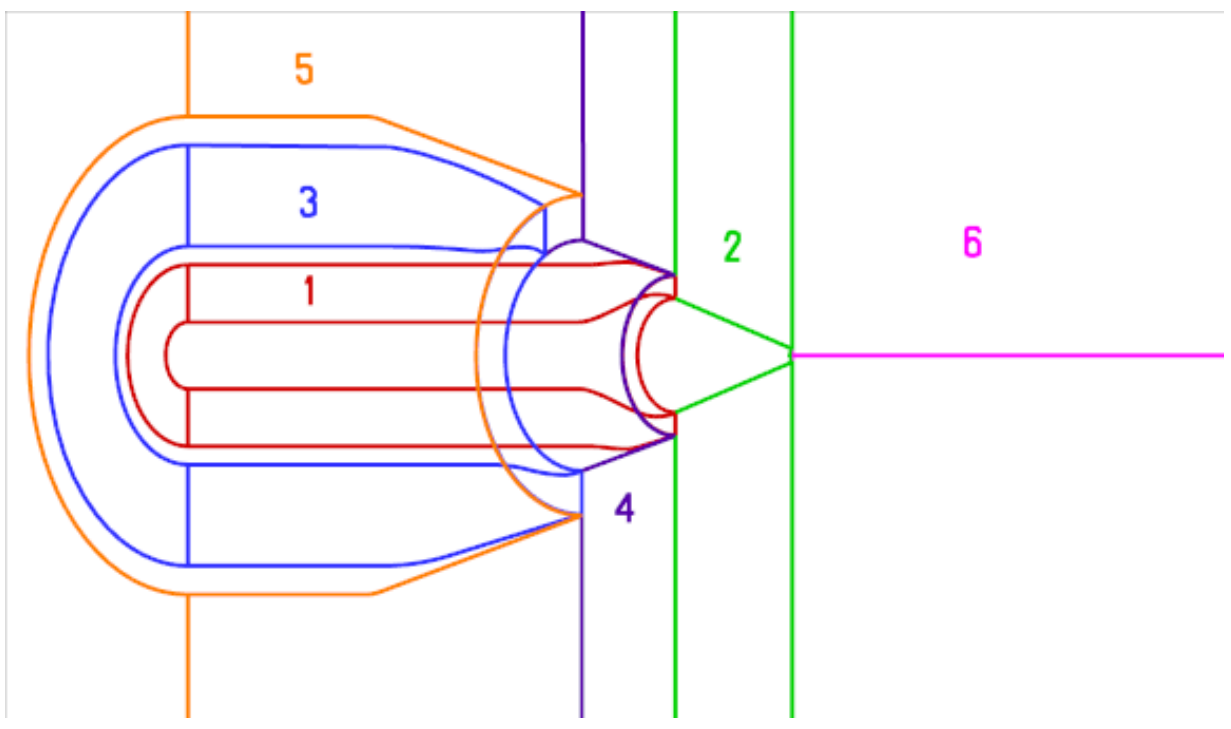

Figure 4. Grid blocking structure 


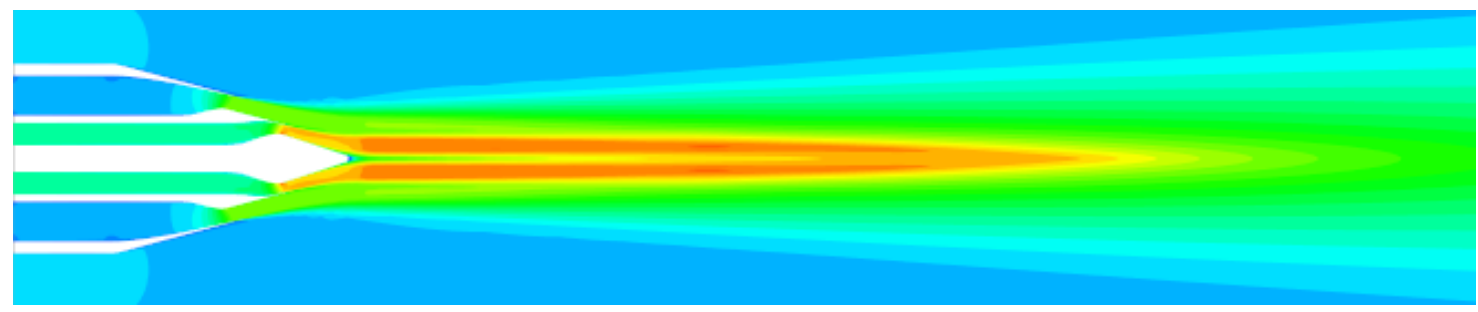

(a) baseline

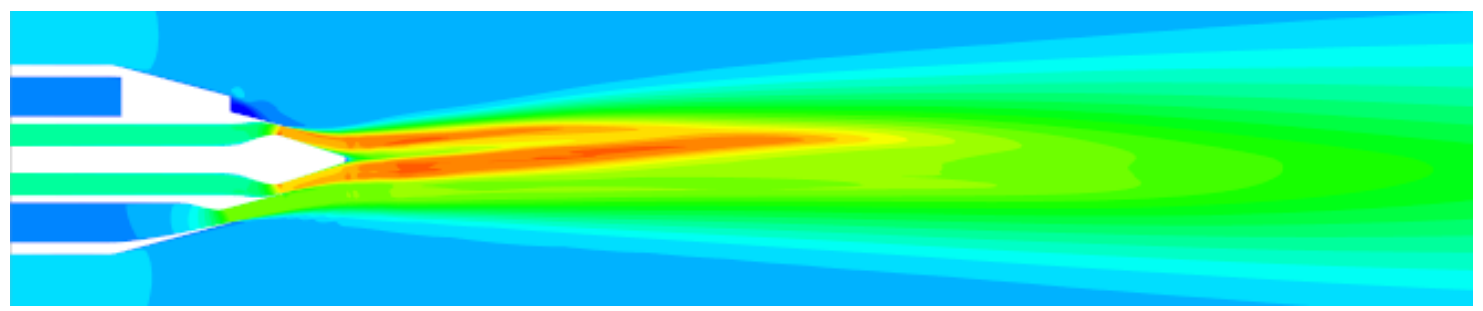

(b) wedge

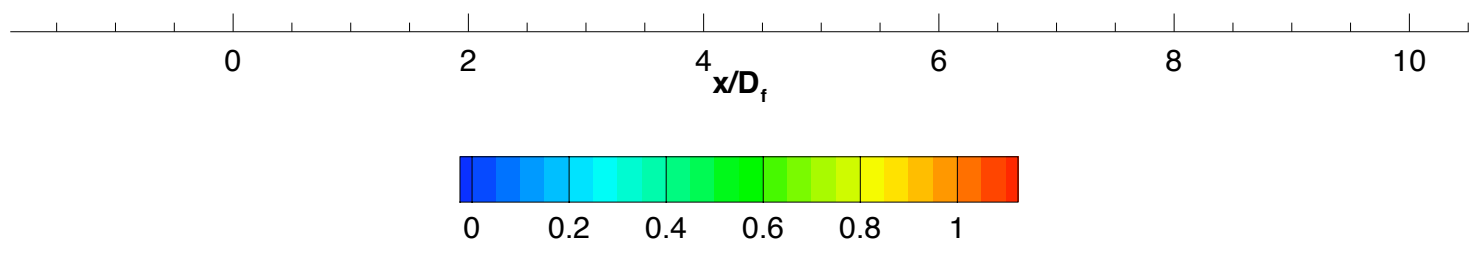

Figure 5. Streamwise (xy-plane) contours of u-velocity, $u / U_{c}$, for 3 BB nozzles

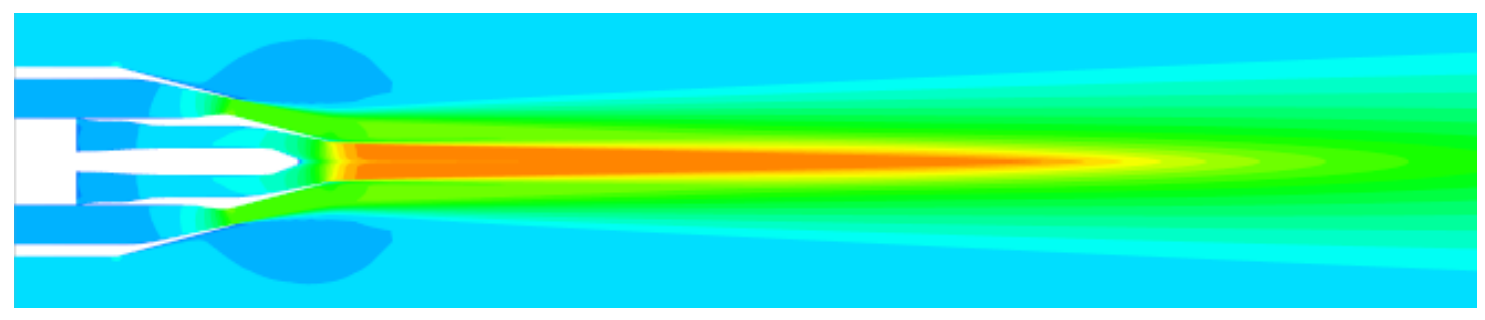

(a) baseline

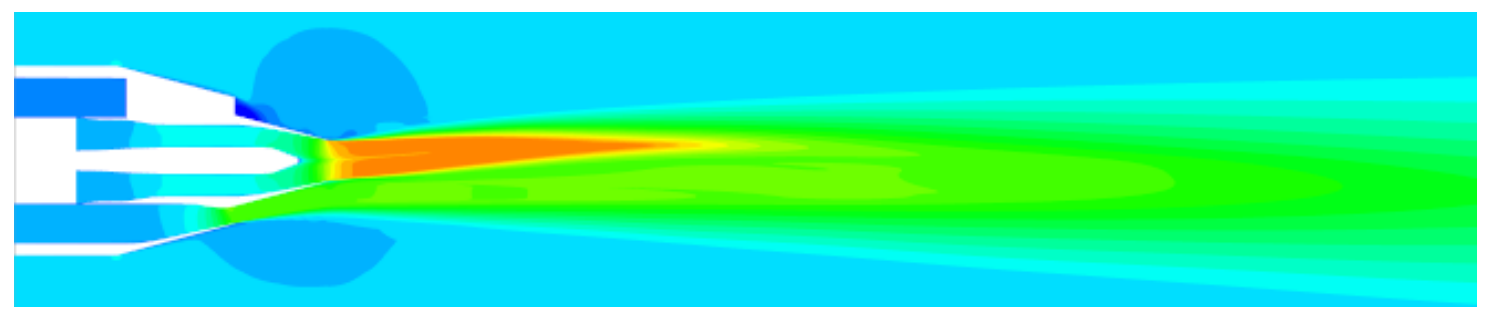

(b) wedge

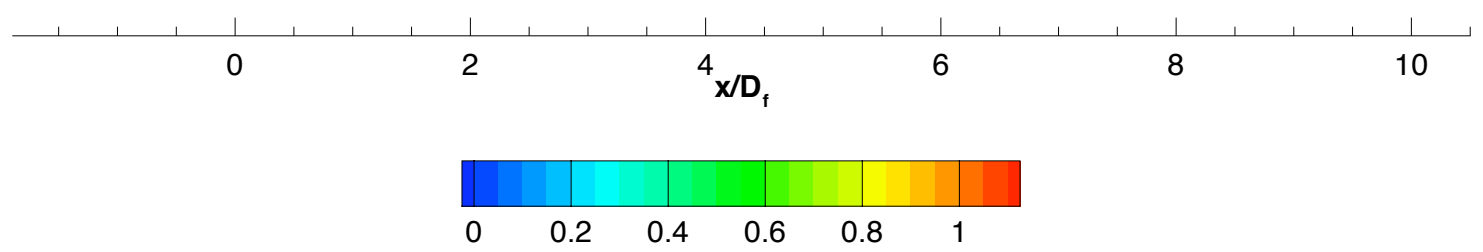

Figure 6. Streamwise (xy-plane) contours of u-velocity, $u / U_{c}$, for $4 \mathrm{BB}$ nozzles 


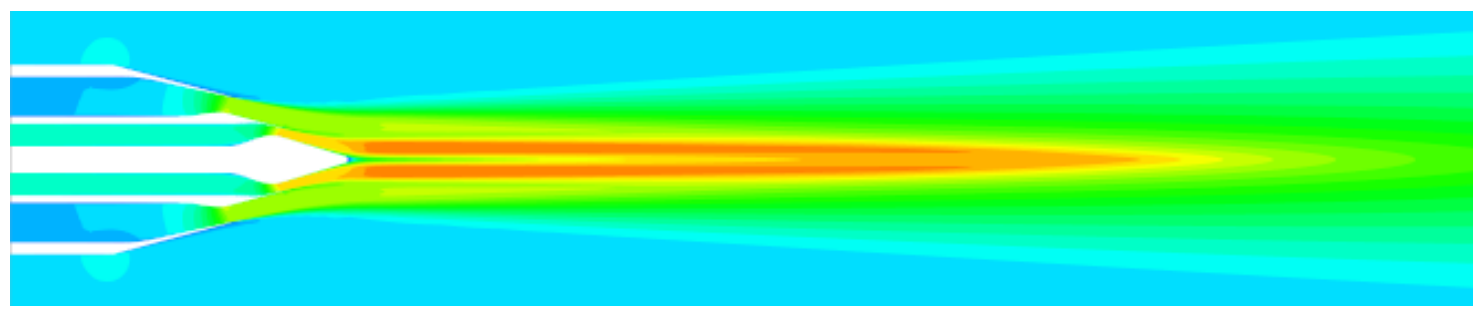

(a) baseline

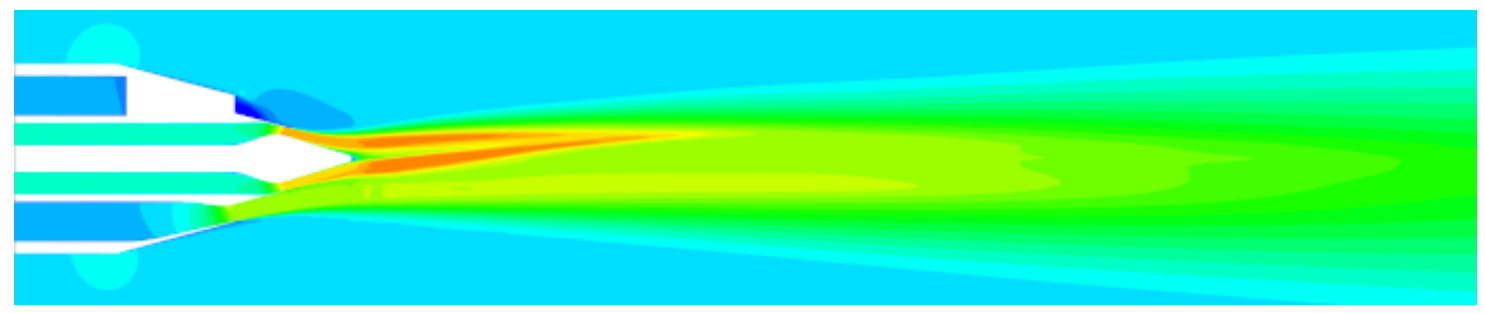

(b) wedge

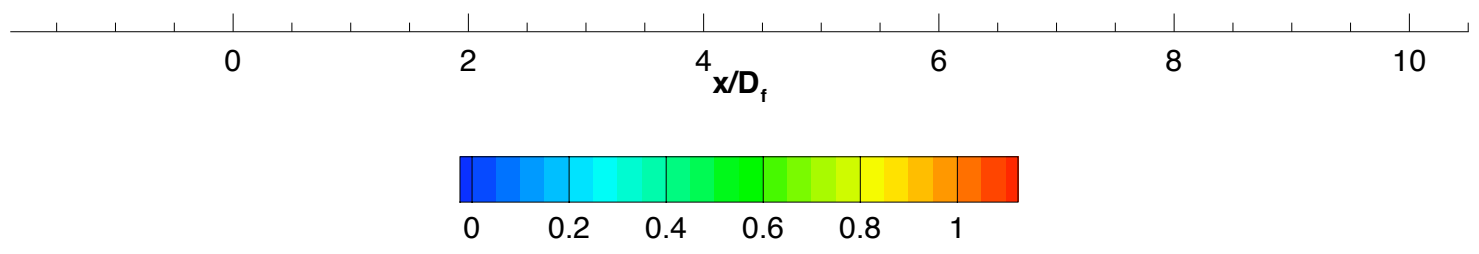

Figure 7. Streamwise (xy-plane) contours of u-velocity, $u / U_{c}$, for 5BB nozzles

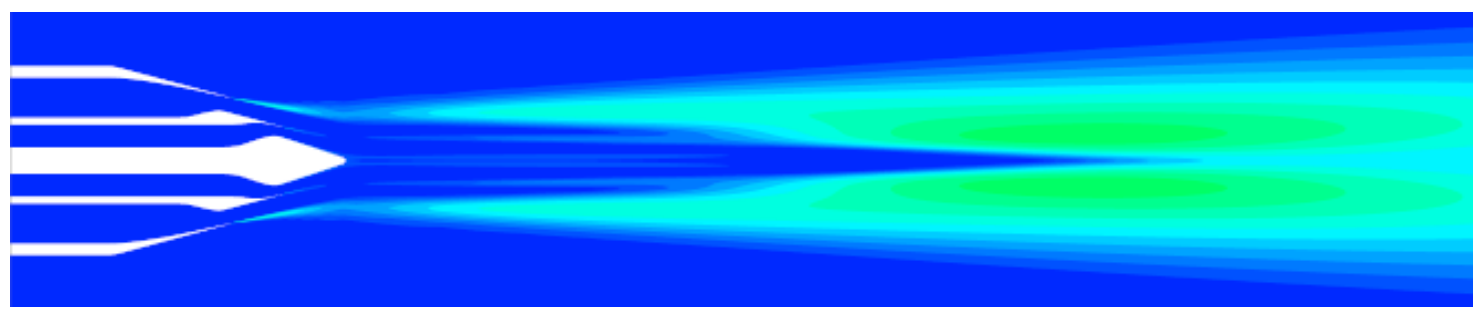

(a) baseline

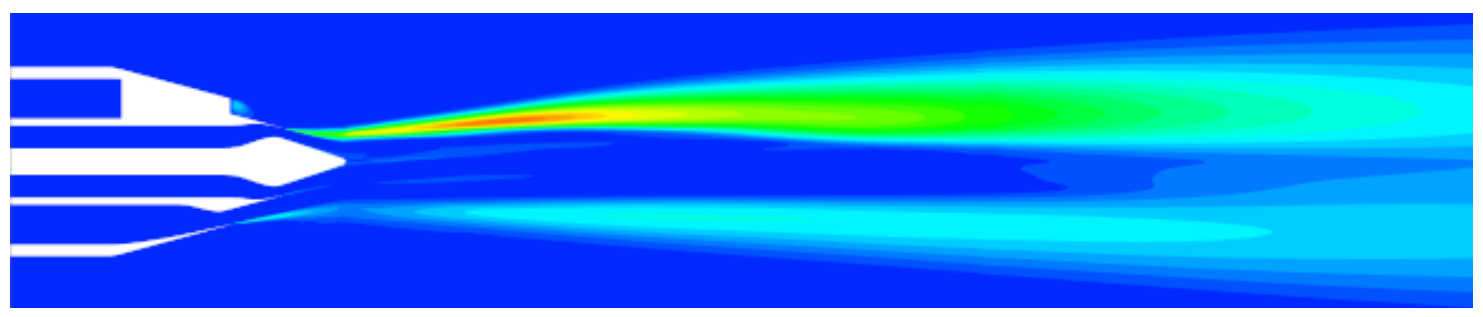

(b) wedge

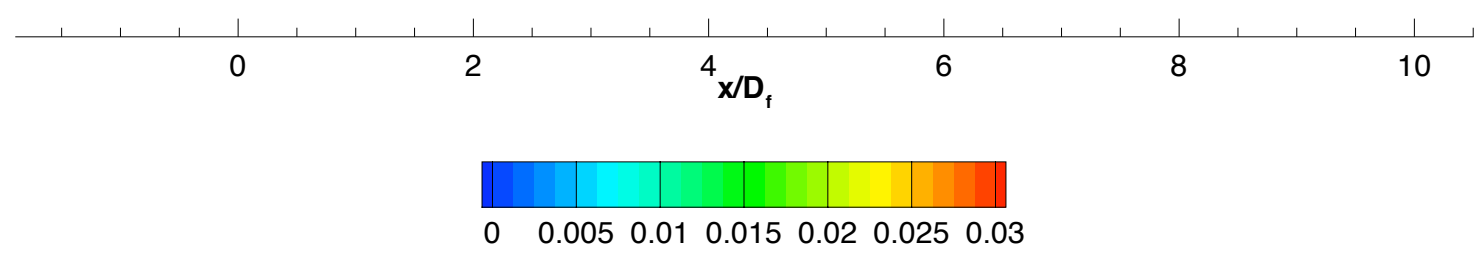

Figure 8. Streamwise (xy-plane) contours of turbulent kinetic energy, $k / U_{c}^{2}$, for 3BB nozzles 


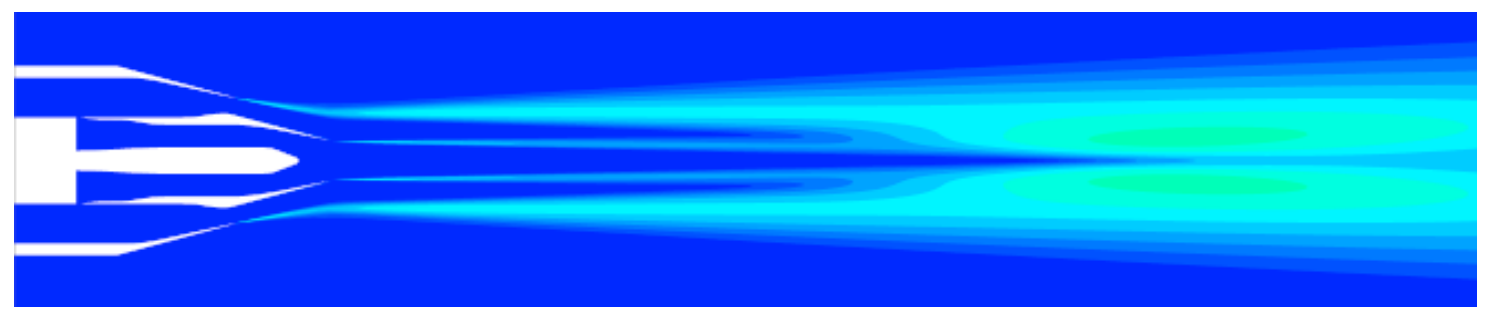

(a) baseline

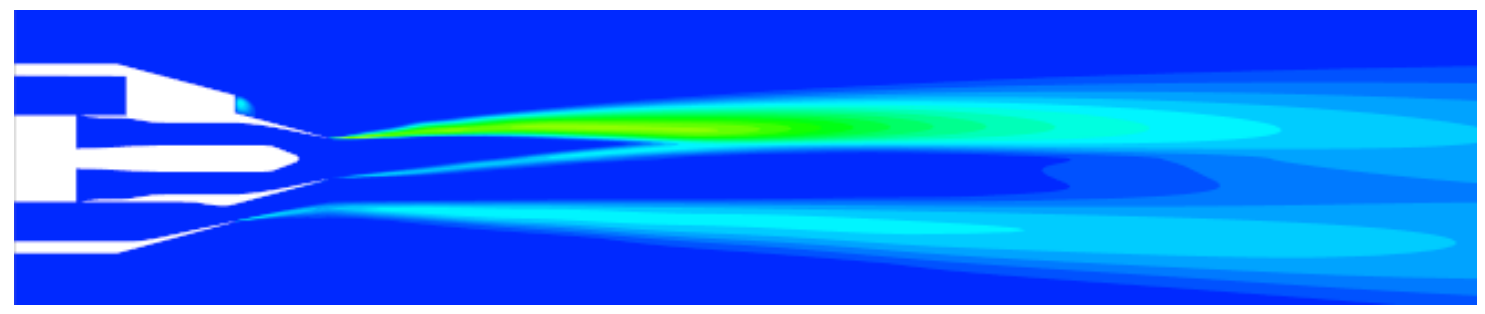

(b) wedge
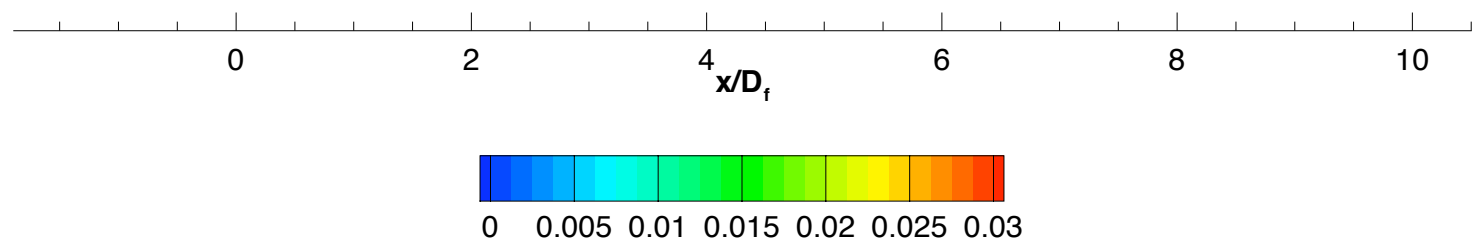

Figure 9. Streamwise (xy-plane) contours of turbulent kinetic energy, $k / U_{c}^{2}$, for $4 \mathrm{BB}$ nozzles

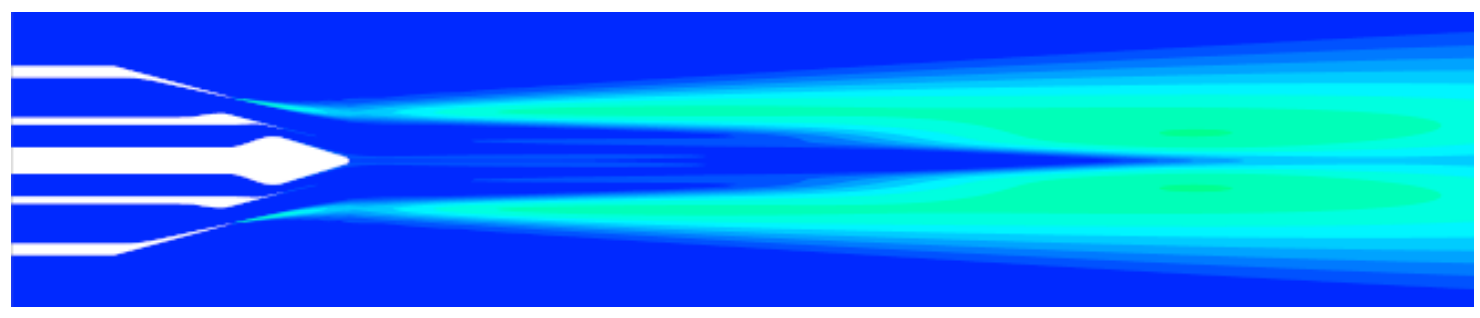

(a) baseline

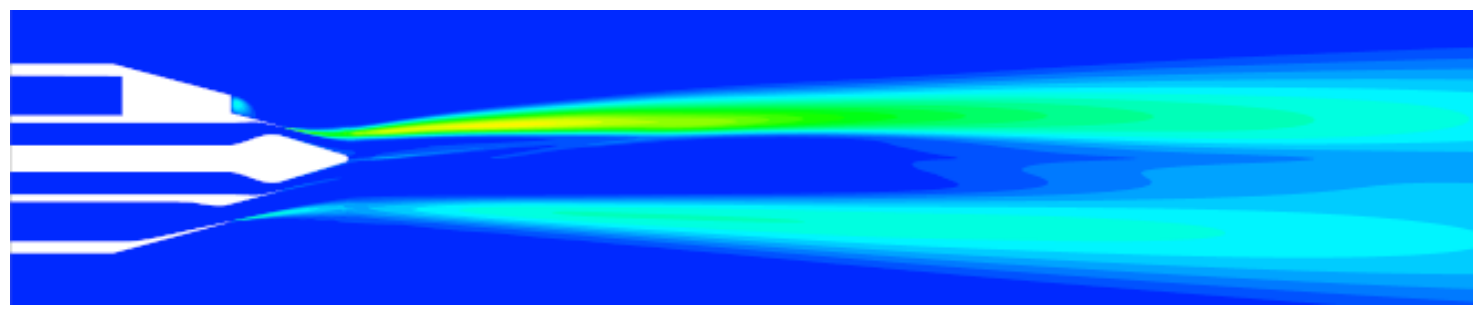

(b) wedge
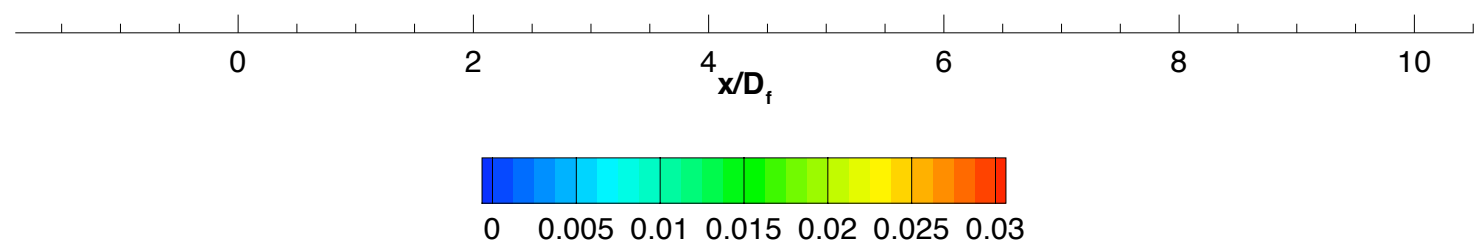

Figure 10. Streamwise (xy-plane) contours of turbulent kinetic energy, $k / U_{c}^{2}$, for 5BB nozzles 


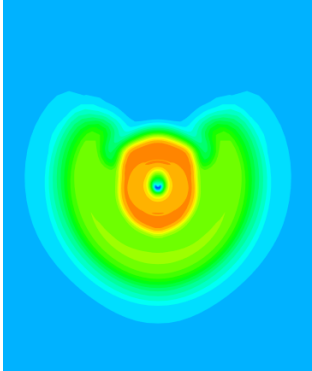

(a) $x / D_{f}=1.0$

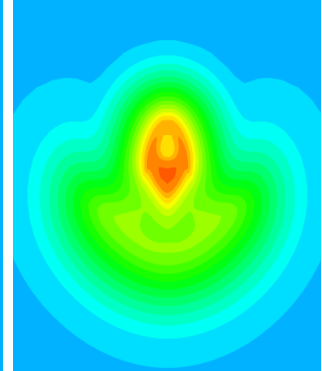

(b) $x / D_{f}=3.0$

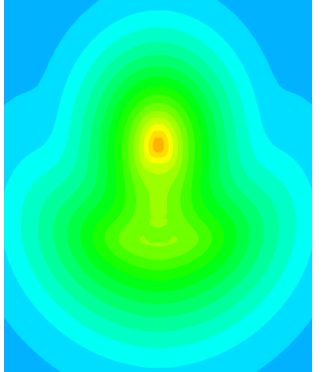

(c) $x / D_{f}=5.0$

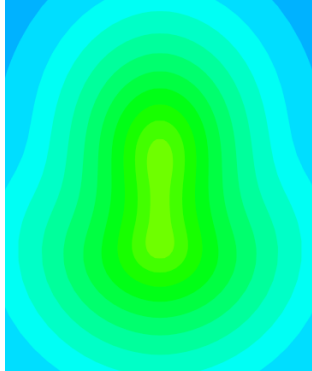

(d) $x / D_{f}=7.0$

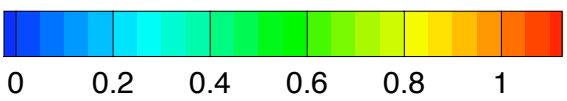

Figure 11. Cross-plane (yz-plane) contours of u-velocity, $u / U_{c}$, for 3 BB nozzles

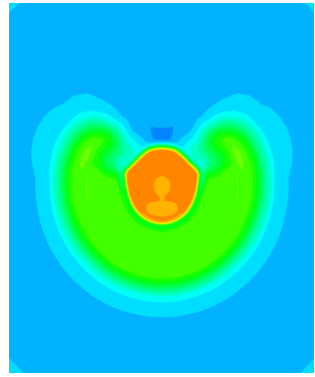

(a) $x / D_{f}=1.0$

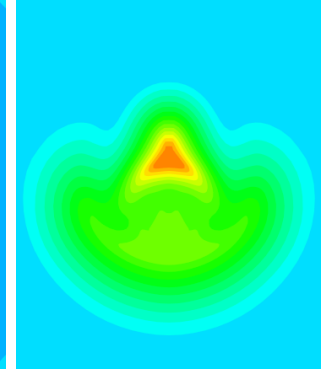

(b) $x / D_{f}=3.0$

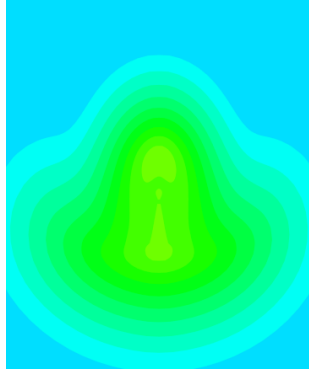

(c) $x / D_{f}=5.0$

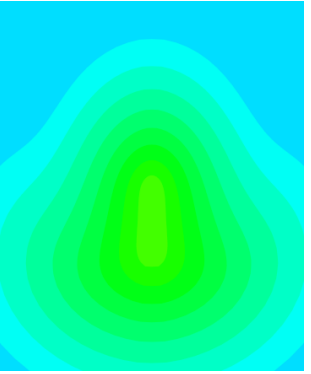

(d) $x / D_{f}=7.0$

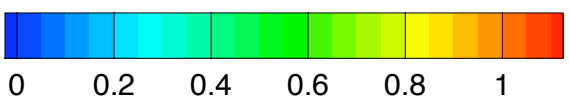

Figure 12. Cross-plane (yz-plane) contours of u-velocity, $u / U_{c}$, for 4BB nozzles

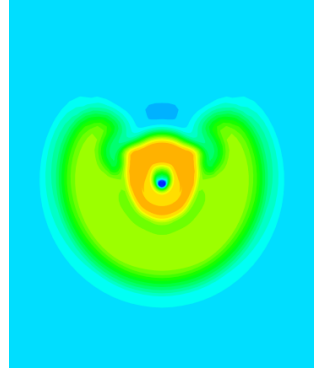

(a) $x / D_{f}=1.0$

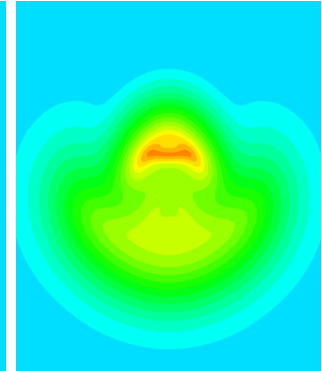

(b) $x / D_{f}=3.0$

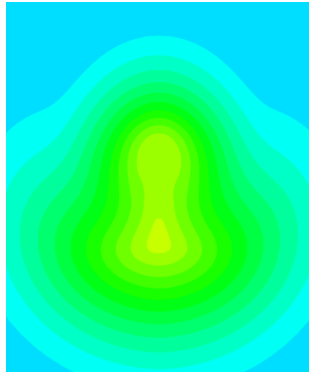

(c) $x / D_{f}=5.0$

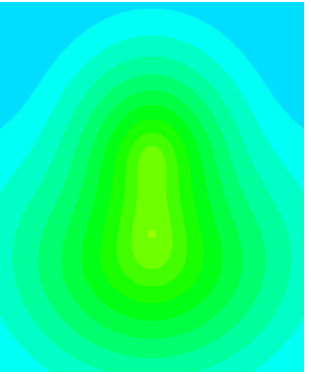

(d) $x / D_{f}=7.0$

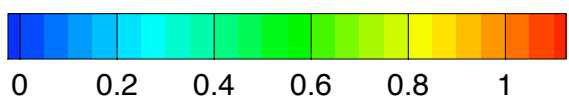

Figure 13. Cross-plane (yz-plane) contours of u-velocity, $u / U_{c}$, for 5BB nozzles 


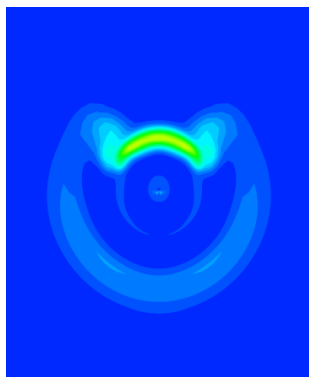

(a) $x / D_{f}=1.0$

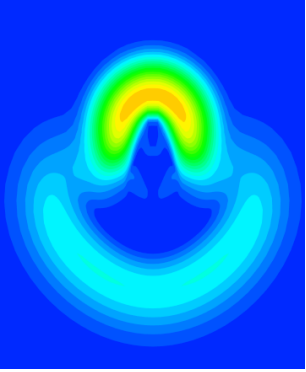

(b) $x / D_{f}=3.0$

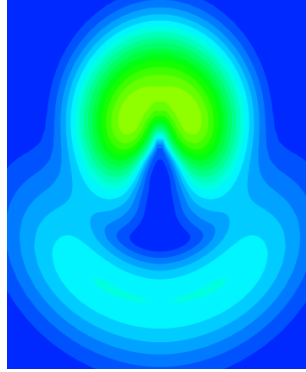

(c) $x / D_{f}=5.0$

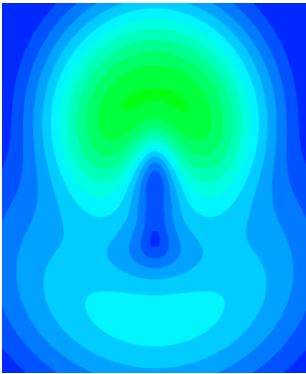

(d) $x / D_{f}=7.0$

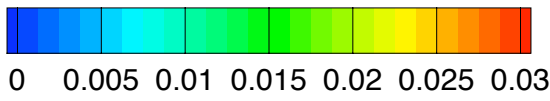

Figure 14. Cross-plane (yz-plane) contours of turbulent kinetic energy, $k / U_{c}^{2}$, for $3 B$ B nozzles

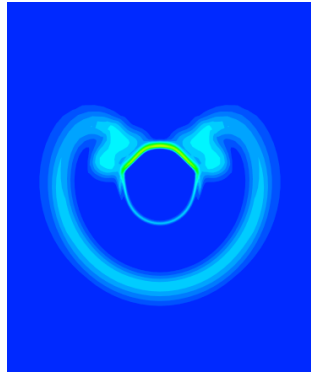

(a) $x / D_{f}=1.0$

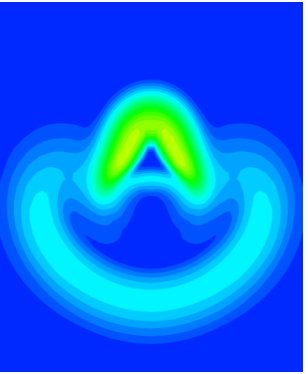

(b) $x / D_{f}=3.0$

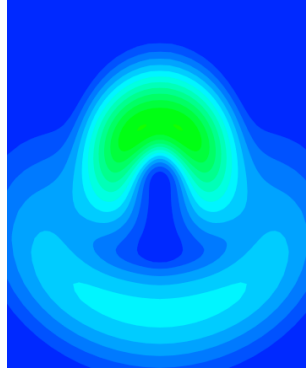

(c) $x / D_{f}=5.0$

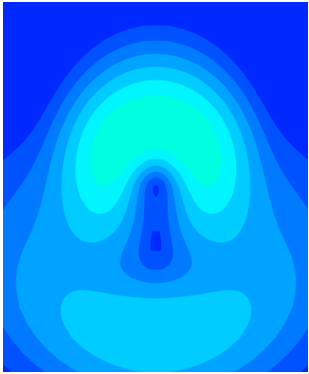

(d) $x / D_{f}=7.0$

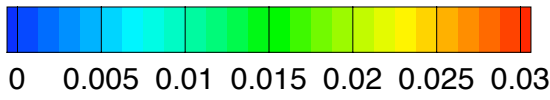

Figure 15. Cross-plane (yz-plane) contours of turbulent kinetic energy, $k / U_{c}^{2}$, for $4 \mathrm{BB}$ nozzles

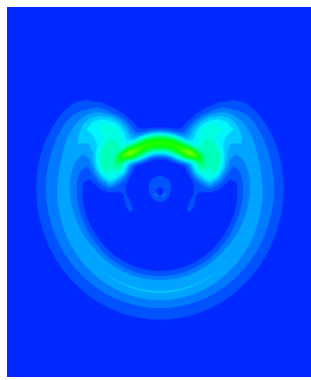

(a) $x / D_{f}=1.0$

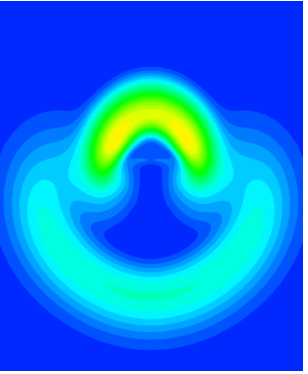

(b) $x / D_{f}=3.0$

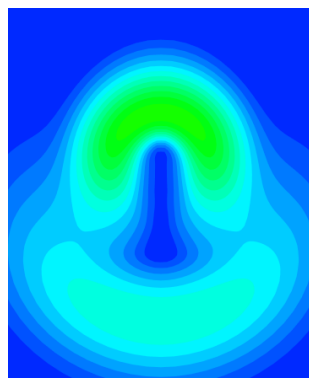

(c) $x / D_{f}=5.0$

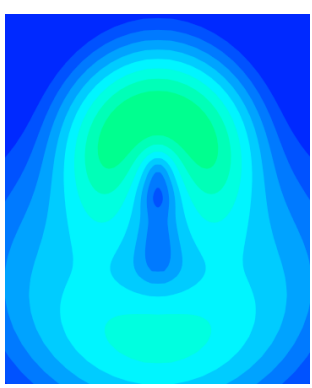

(d) $x / D_{f}=7.0$

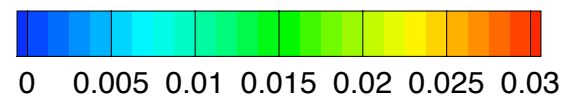

Figure 16. Cross-plane (yz-plane) contours of turbulent kinetic energy, $k / U_{c}^{2}$, for 5BB nozzles 


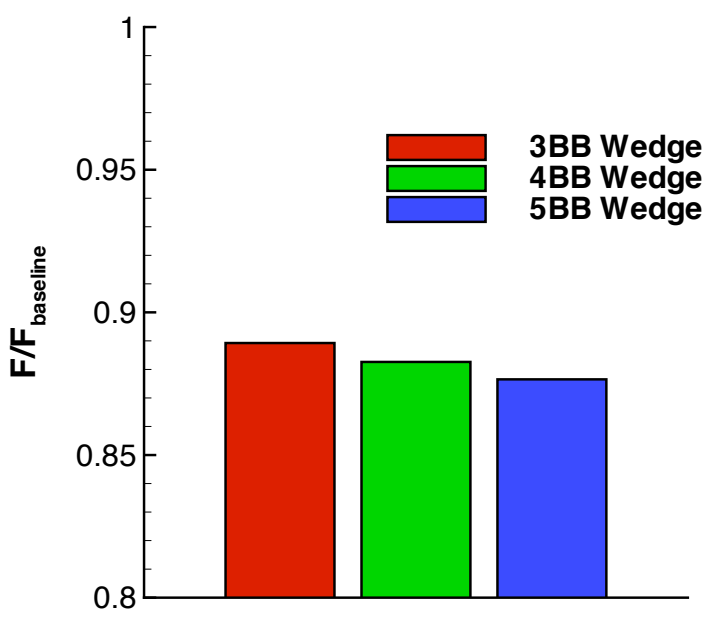

(a) thrust

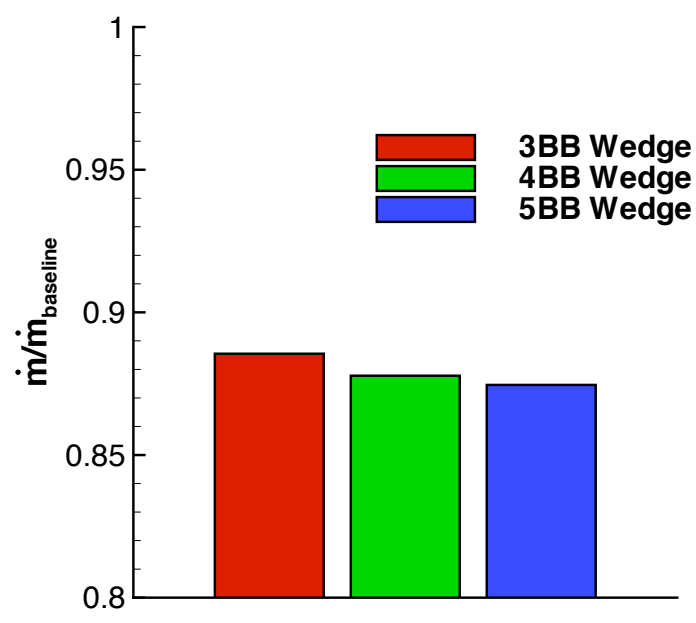

(b) massflow

Figure 17. Ratio of wedge nozzle to baseline nozzle performance

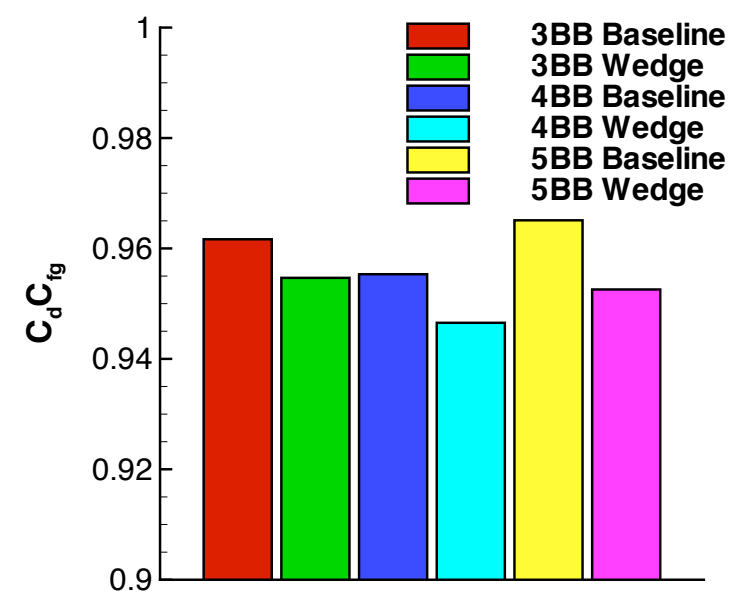

(a) Performance of baseline and wedge nozzles

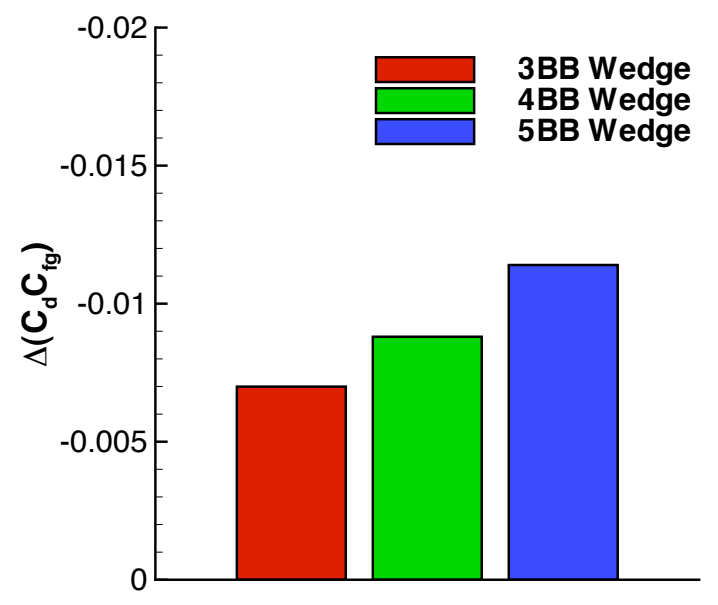

(b) Effect of wedge on nozzle performance

Figure 18. Nozzle performance expressed as thrust coefficient 


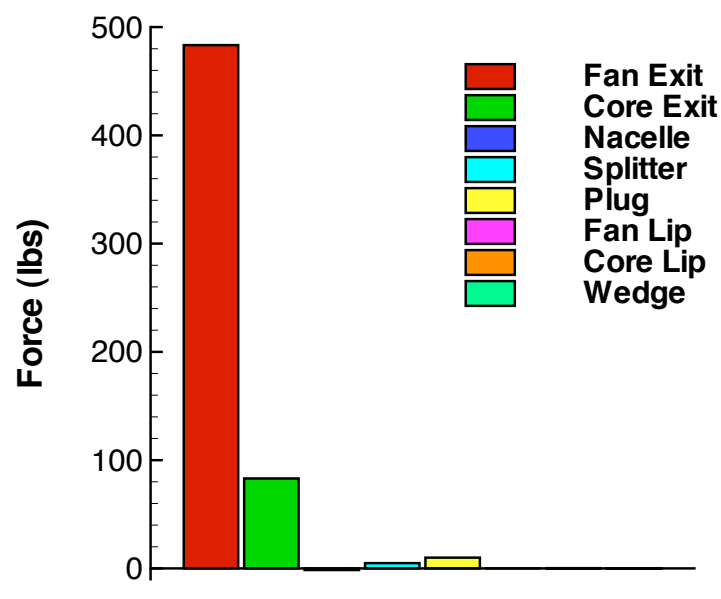

(a) Baseline

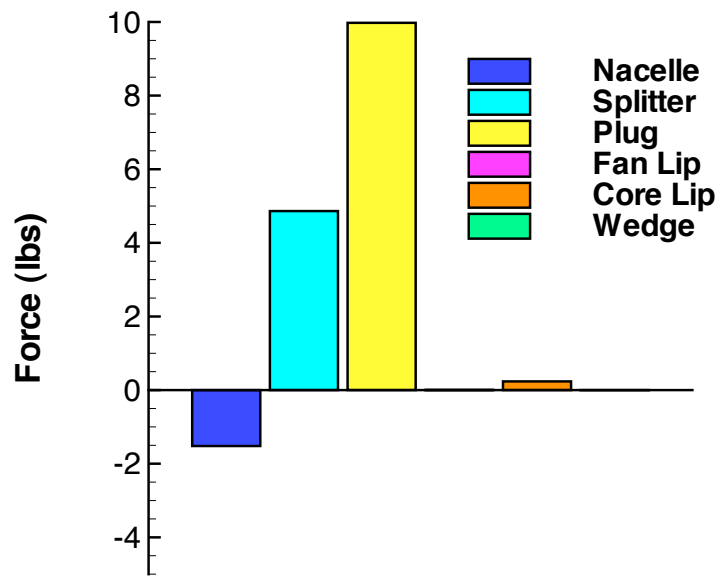

(c) Baseline detail

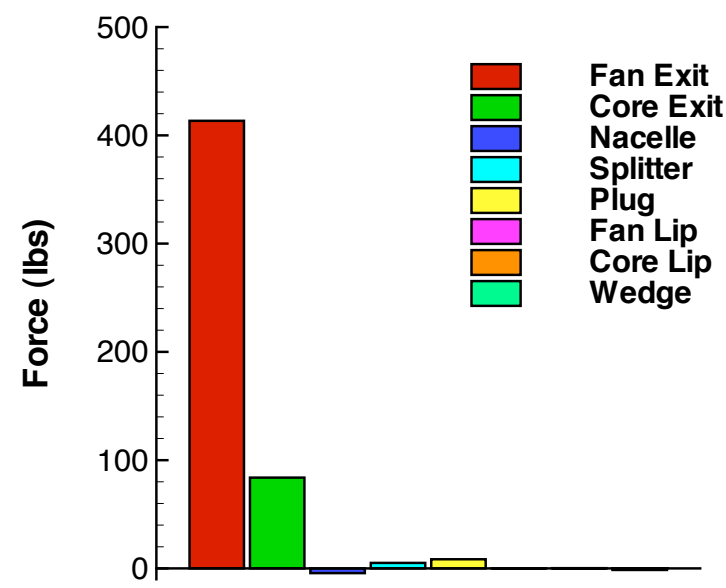

(b) Wedge

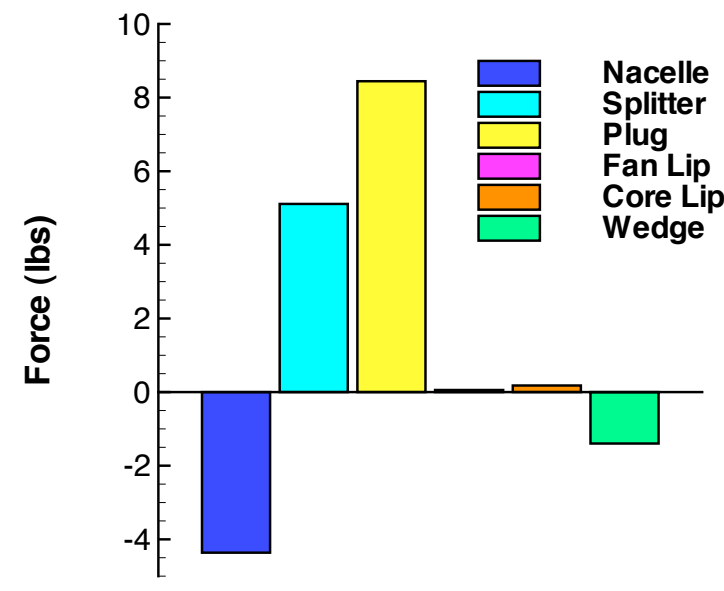

(d) Wedge detail

Figure 19. Force breakout for 5BB baseline and wedge nozzles 


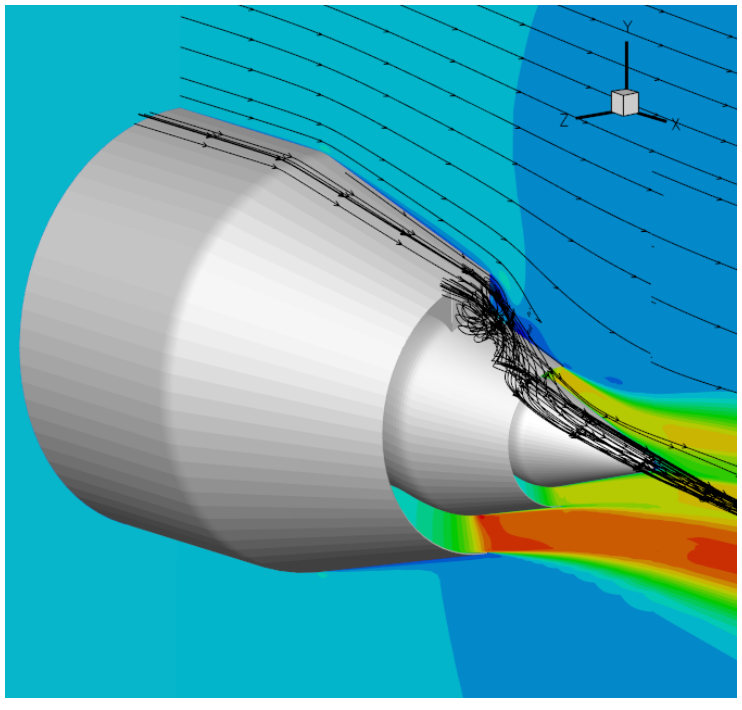

(a) External streamlines

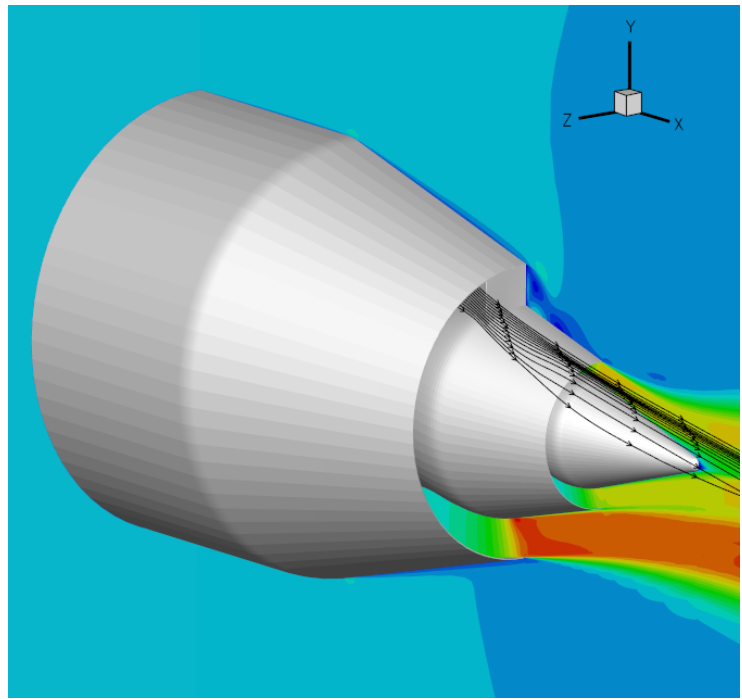

(b) Internal streamlines

Figure 20. Streamlines for the 5BB nozzle

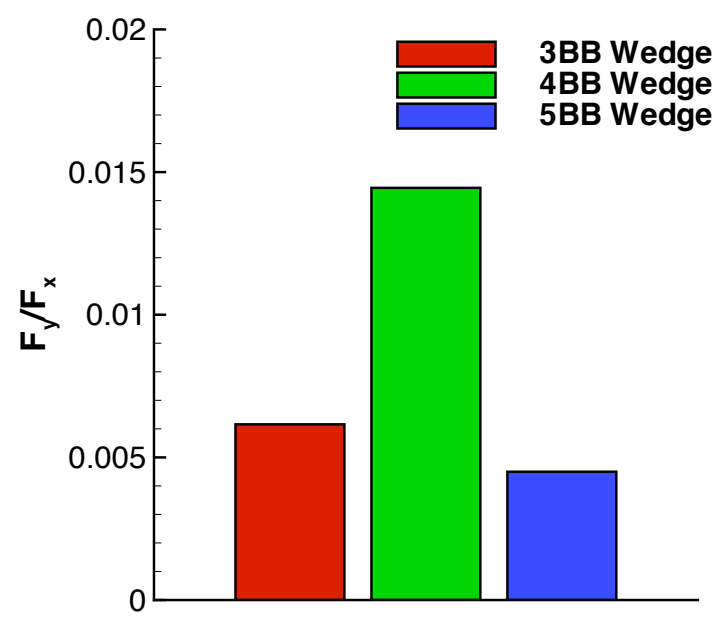

Figure 21. Component of vertical thrust 


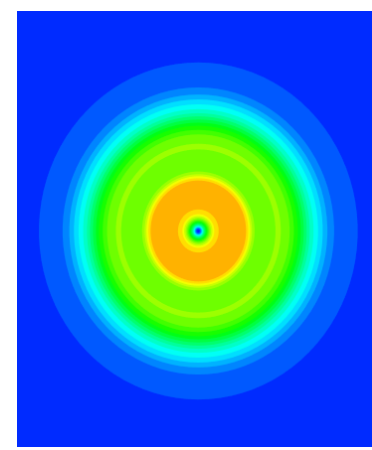

(a) CFD, $x / D_{f}=1.0$

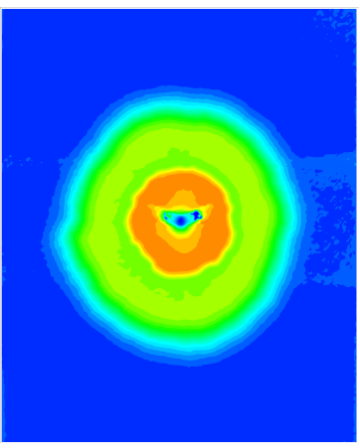

(d) exp., $x / D_{f}=1.0$

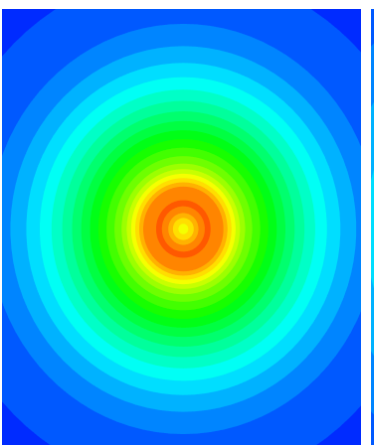

(g) CFD, $x / D_{f}=3.0$

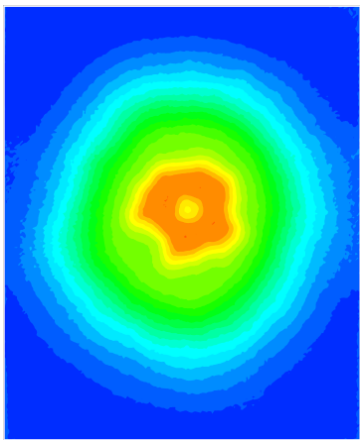

(j) exp., $x / D_{f}=3.0$

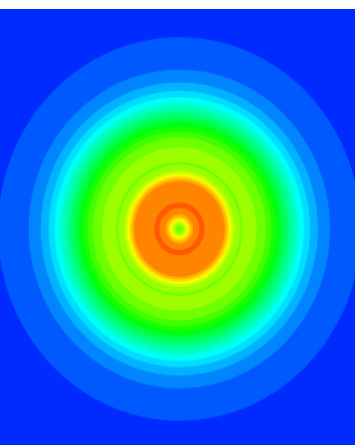

(b) CFD, $x / D_{f}=1.5$

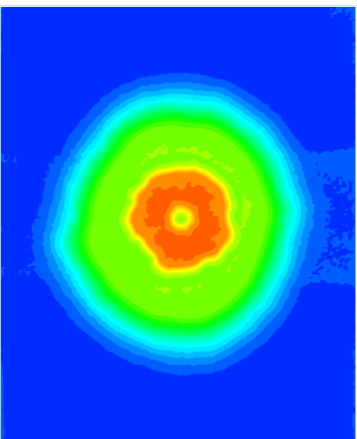

(e) exp., $x / D_{f}=1.5$

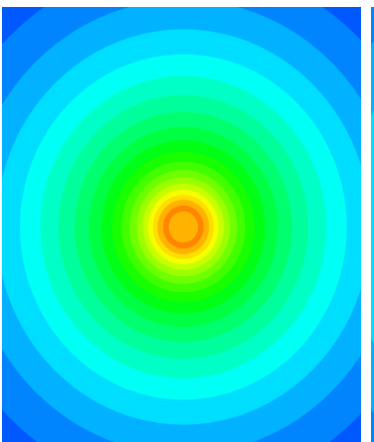

(h) CFD, $x / D_{f}=5.0$

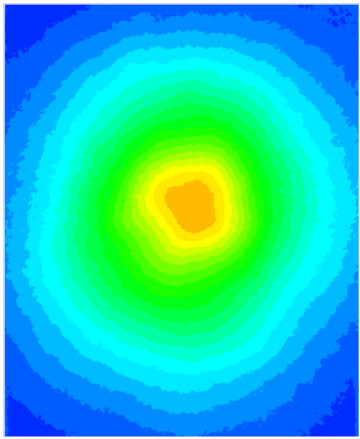

(k) exp., $x / D_{f}=5.0$

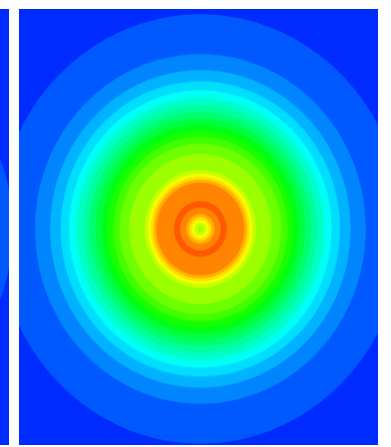

(c) CFD, $x / D_{f}=2.0$

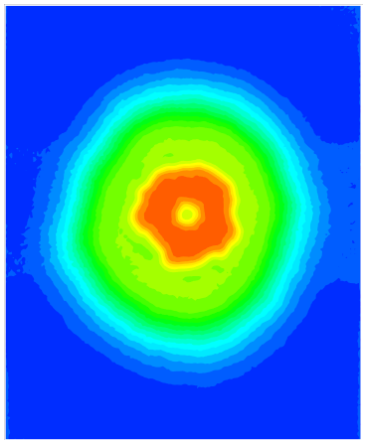

(f) exp., $x / D_{f}=2.0$

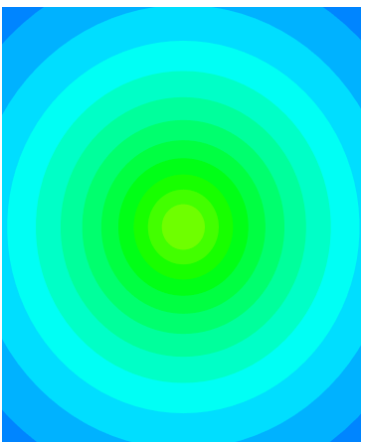

(i) CFD, $x / D_{f}=7.0$

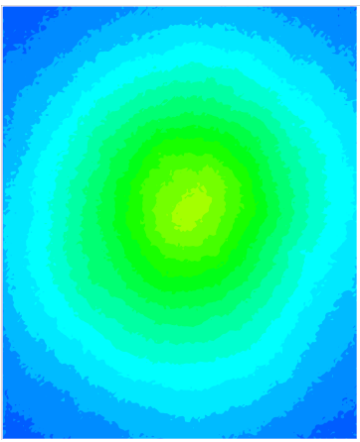

(l) exp., $x / D_{f}=7.0$

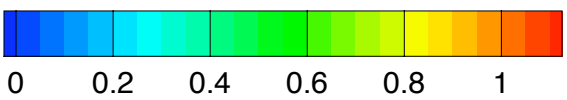

Figure 22. Contours of u-velocity, $u / U_{C}$, for 3BB baseline nozzle (no wedge), hot conditions 


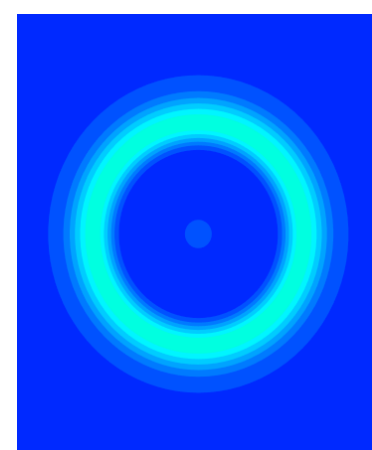

(a) CFD, $x / D_{f}=1.0$

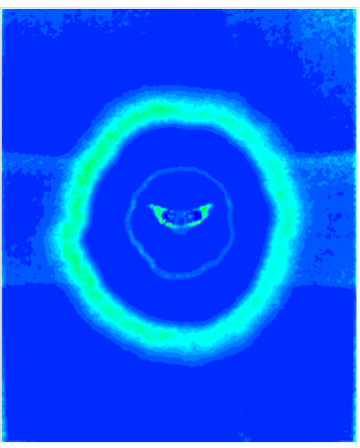

(d) exp., $x / D_{f}=1.0$

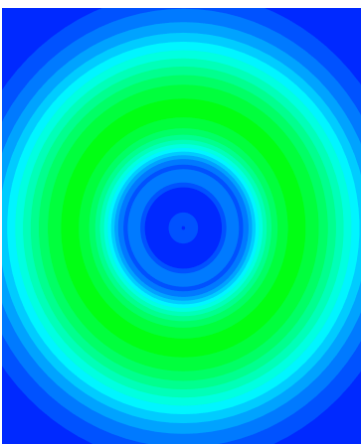

(g) CFD, $x / D_{f}=3.0$

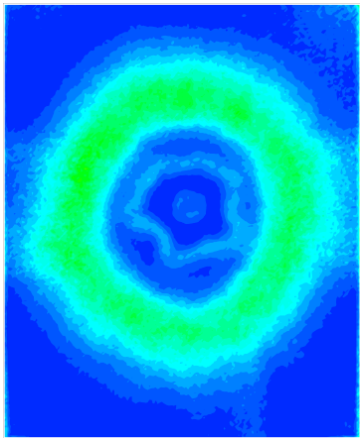

(j) exp., $x / D_{f}=3.0$

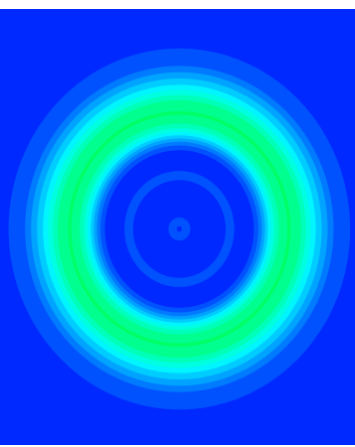

(b) CFD, $x / D_{f}=1.5$

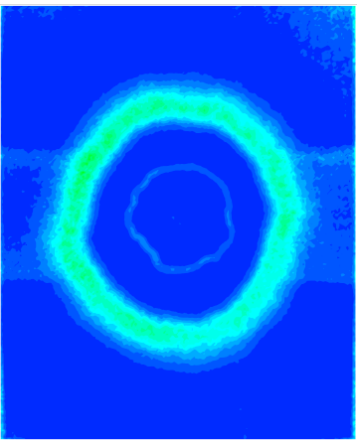

(e) exp., $x / D_{f}=1.5$

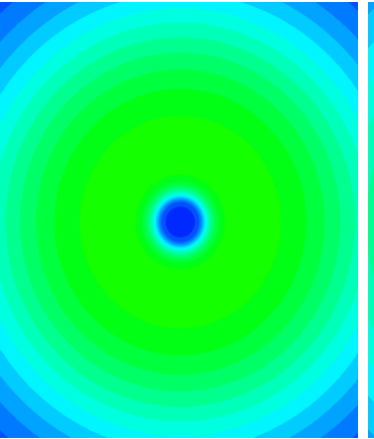

(h) CFD, $x / D_{f}=5.0$

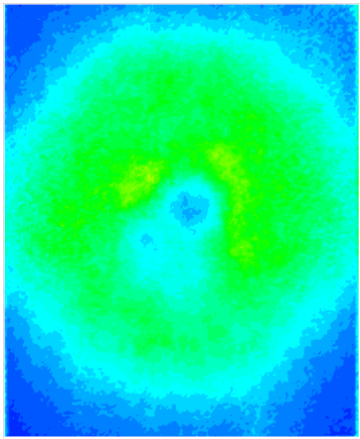

(k) exp., $x / D_{f}=5.0$

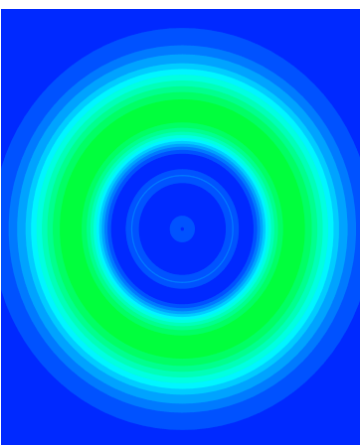

(c) CFD, $x / D_{f}=2.0$

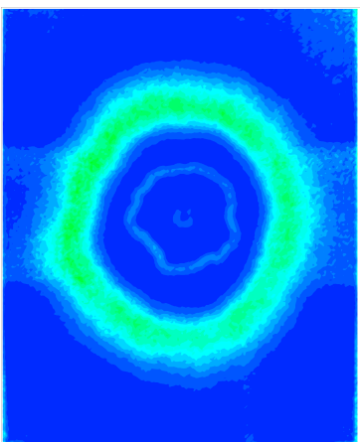

(f) exp., $x / D_{f}=2.0$

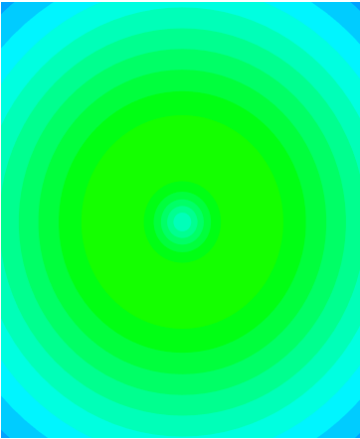

(i) CFD, $x / D_{f}=7.0$

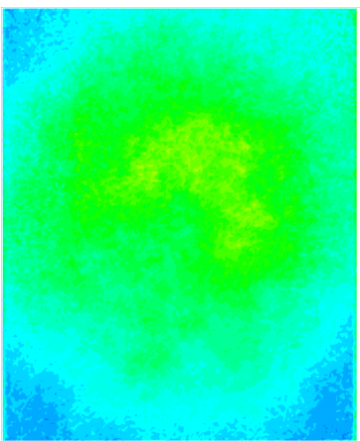

(l) exp., $x / D_{f}=7.0$

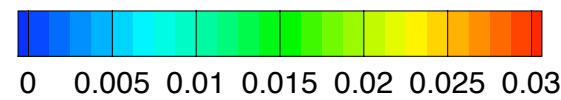

Figure 23. Contours of turbulent kinetic energy, $k / U_{c}^{2}$, for $3 B \mathrm{~B}$ baseline nozzle (no wedge), hot conditions 


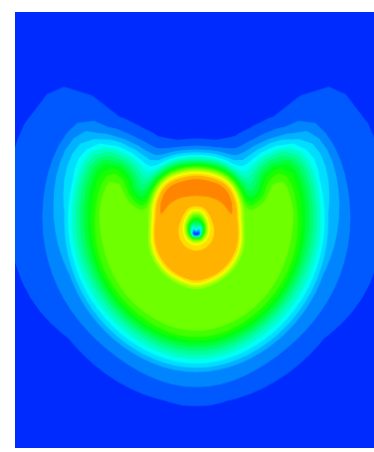

(a) CFD, $x / D_{f}=1.0$

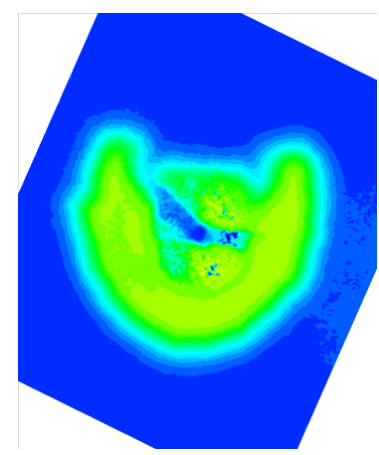

(d) exp., $x / D_{f}=1.0$

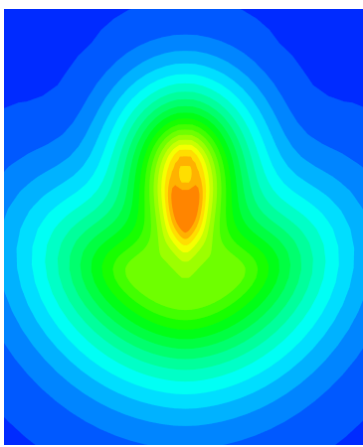

(g) CFD, $x / D_{f}=3.0$

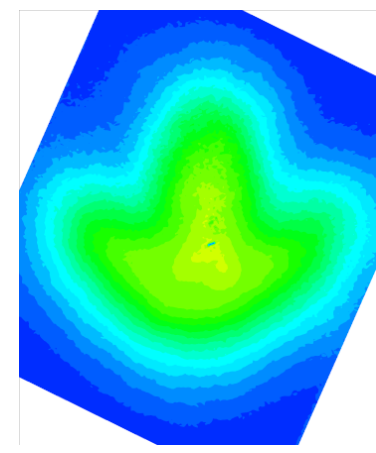

(j) exp., $x / D_{f}=3.0$

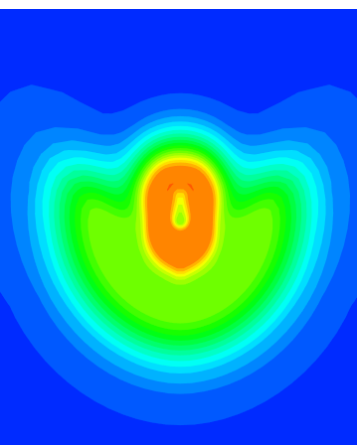

(b) CFD, $x / D_{f}=1.5$

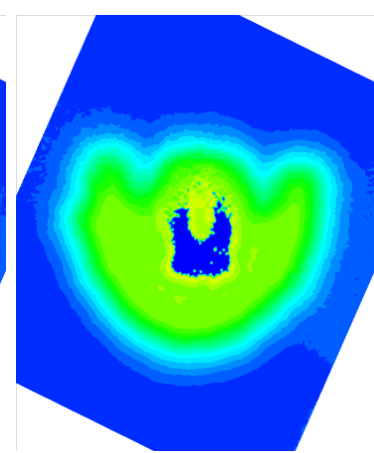

(e) exp., $x / D_{f}=1.5$

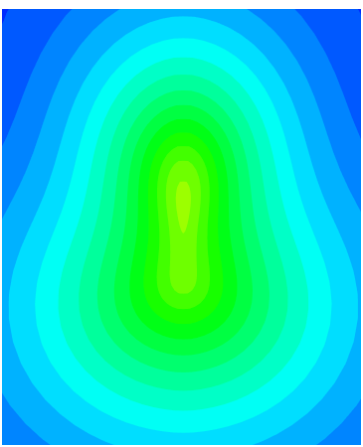

(h) CFD, $x / D_{f}=5.0$

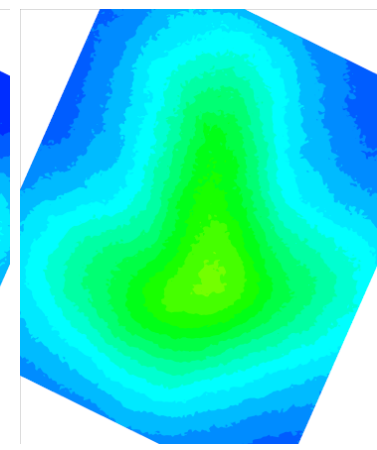

(k) exp., $x / D_{f}=5.0$

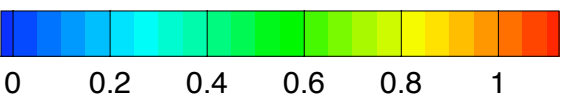

Figure 24. Contours of u-velocity, $u / U_{c}$, for 3 BB nozzle with wedge, hot conditions

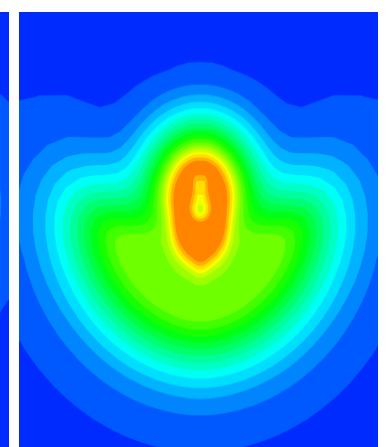

(c) $\mathrm{CFD}, x / D_{f}=2.0$

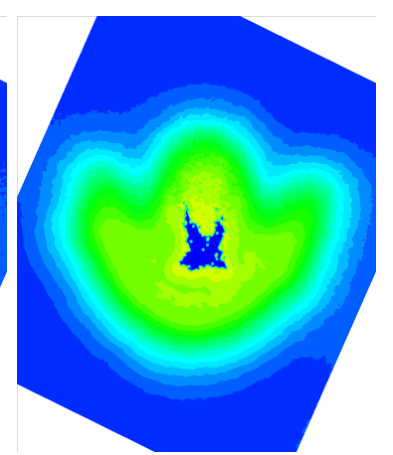

(f) exp., $x / D_{f}=2.0$

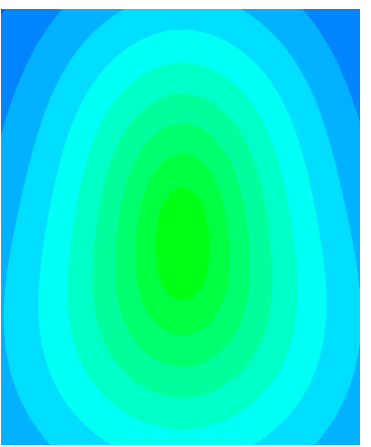

(i) $\mathrm{CFD}, x / D_{f}=7.0$

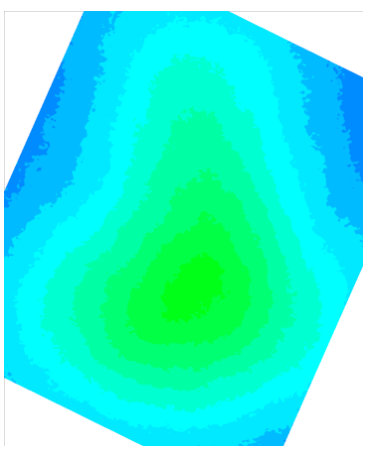

(l) exp., $x / D_{f}=7.0$ 


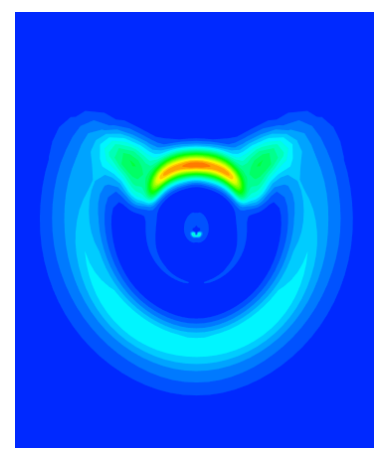

(a) CFD, $x / D_{f}=1.0$

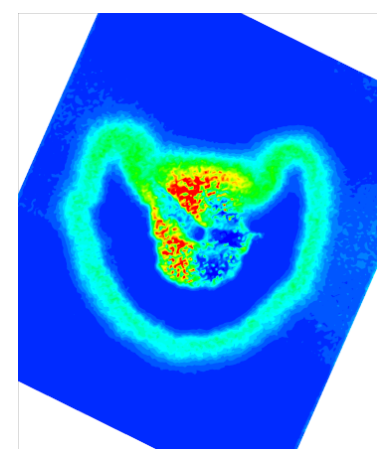

(d) exp., $x / D_{f}=1.0$

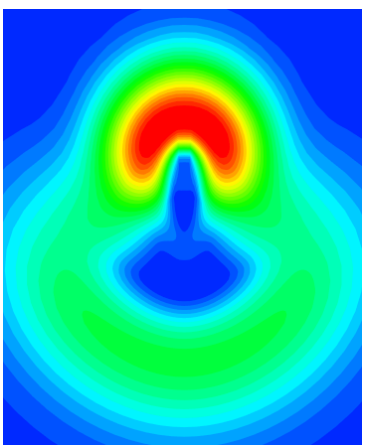

(g) CFD, $x / D_{f}=3.0$

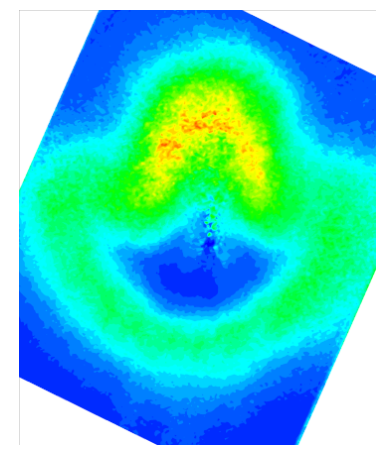

(j) exp., $x / D_{f}=3.0$

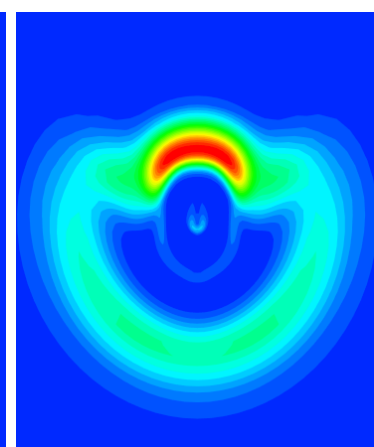

(b) CFD, $x / D_{f}=1.5$

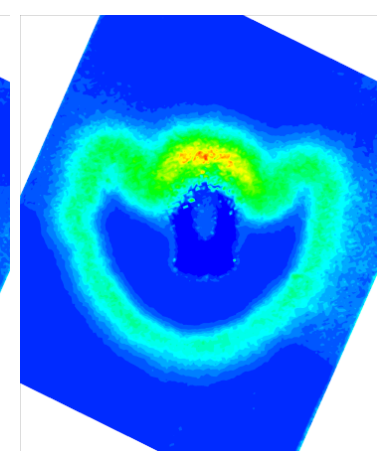

(e) exp., $x / D_{f}=1.5$

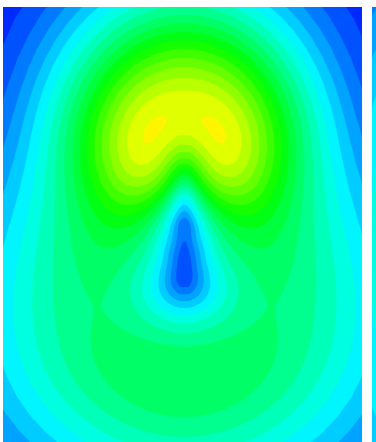

(h) CFD, $x / D_{f}=5.0$

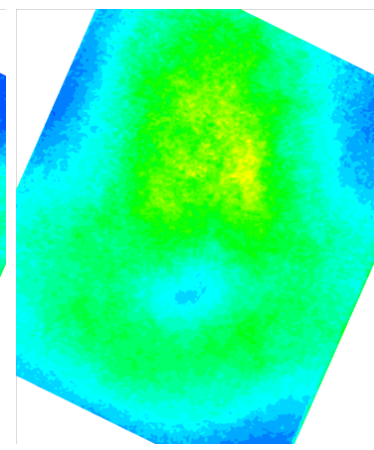

(k) exp., $x / D_{f}=5.0$

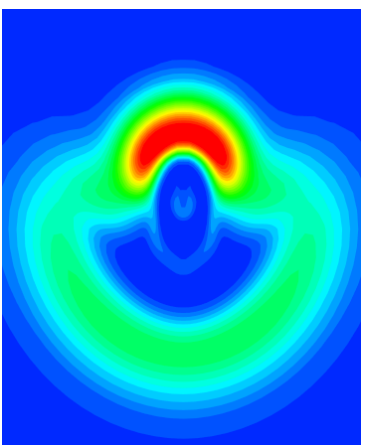

(c) $\mathrm{CFD}, x / D_{f}=2.0$

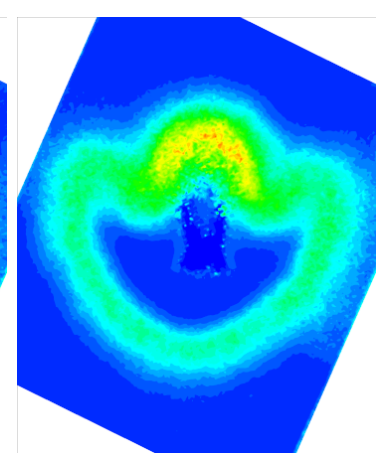

(f) exp., $x / D_{f}=2.0$

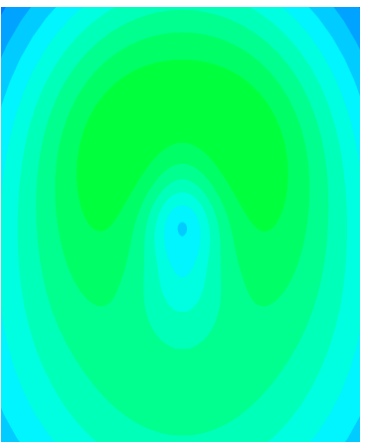

(i) $\mathrm{CFD}, x / D_{f}=7.0$

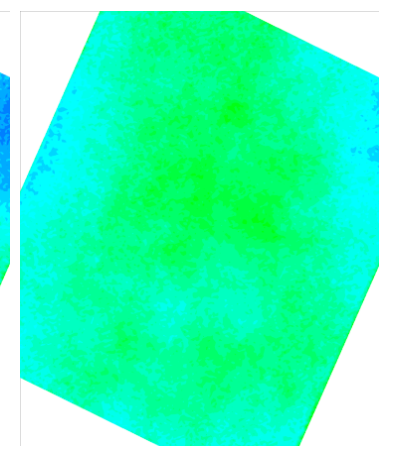

(l) exp., $x / D_{f}=7.0$

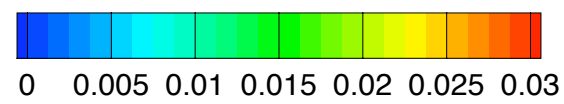

Figure 25. Contours of turbulent kinetic energy, $k / U_{c}^{2}$, for 3BB nozzle with wedge, hot conditions 


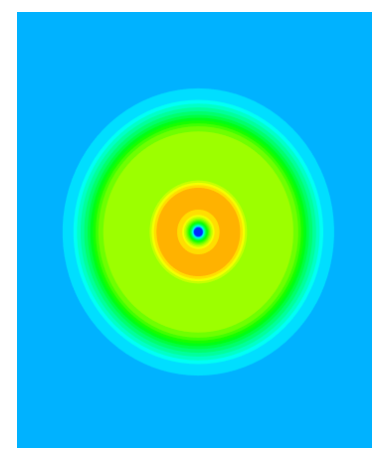

(a) CFD, $x / D_{f}=1.0$

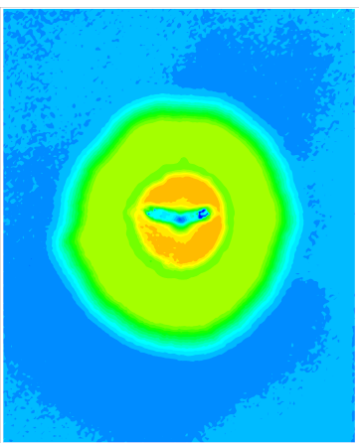

(d) exp., $x / D_{f}=1.0$

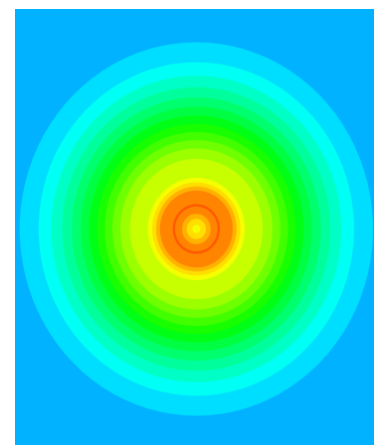

(g) CFD, $x / D_{f}=3.0$

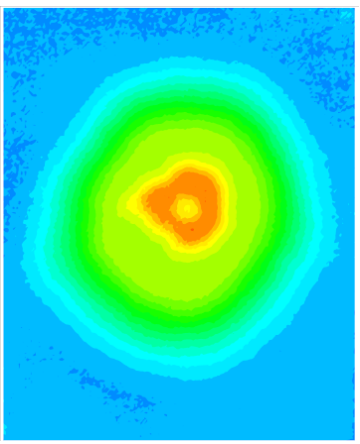

(j) exp., $x / D_{f}=3.0$

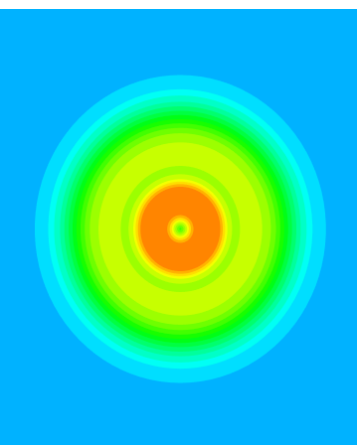

(b) CFD, $x / D_{f}=1.5$

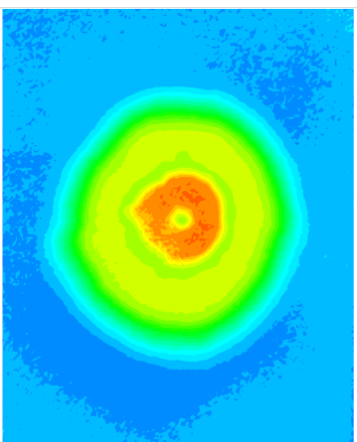

(e) exp., $x / D_{f}=1.5$

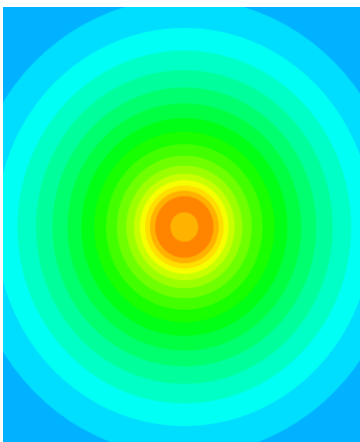

(h) CFD, $x / D_{f}=5.0$

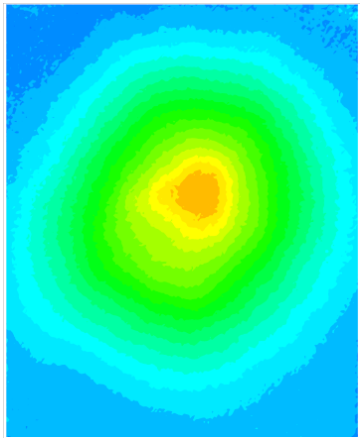

(k) exp., $x / D_{f}=5.0$

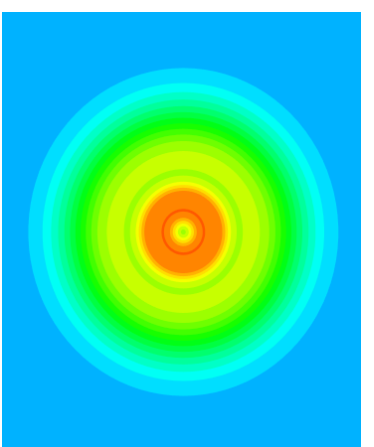

(c) CFD, $x / D_{f}=2.0$

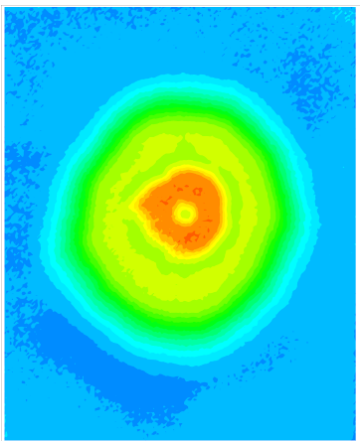

(f) exp., $x / D_{f}=2.0$

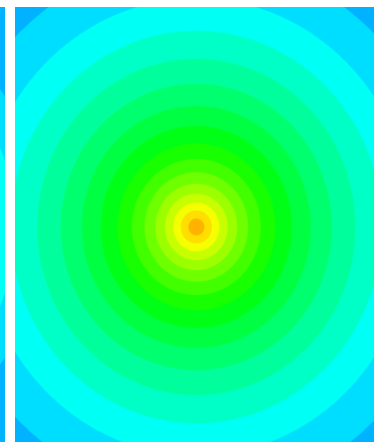

(i) $\mathrm{CFD}, x / D_{f}=7.0$

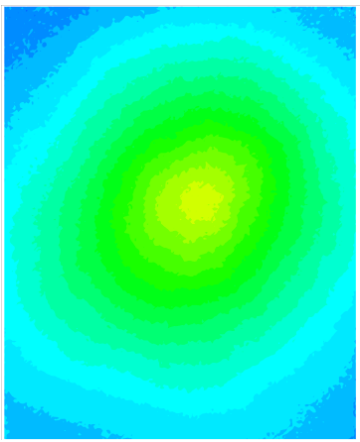

(l) exp., $x / D_{f}=7.0$

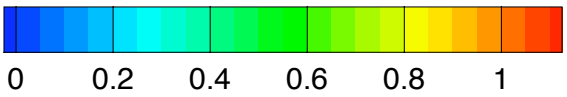

Figure 26. Contours of u-velocity, $u / U_{c}$, for 5BB baseline nozzle (no wedge), hot conditions 


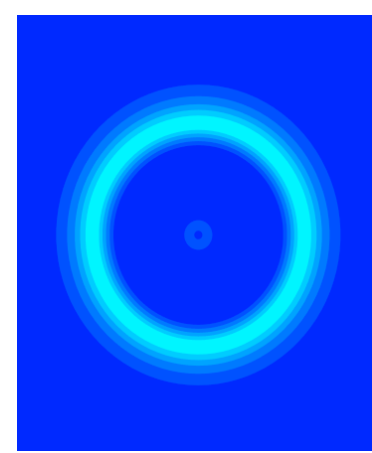

(a) $\mathrm{CFD}, x / D_{f}=1.0$

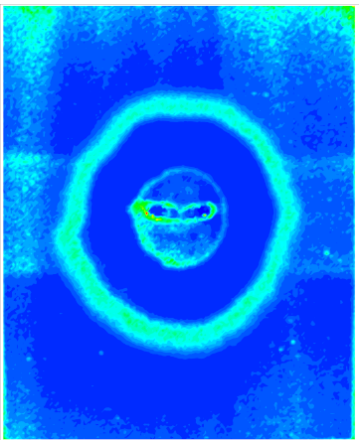

(d) exp., $x / D_{f}=1.0$

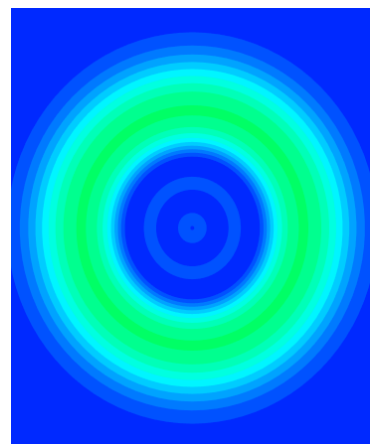

(g) CFD, $x / D_{f}=3.0$

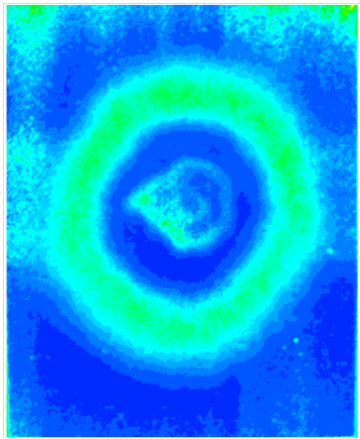

(j) exp., $x / D_{f}=3.0$

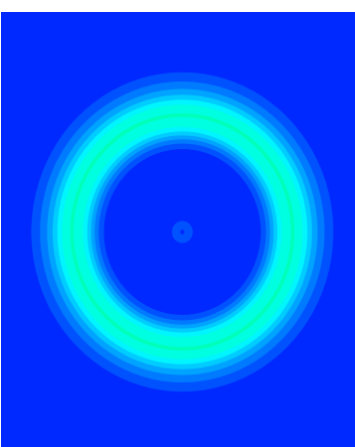

(b) CFD, $x / D_{f}=1.5$

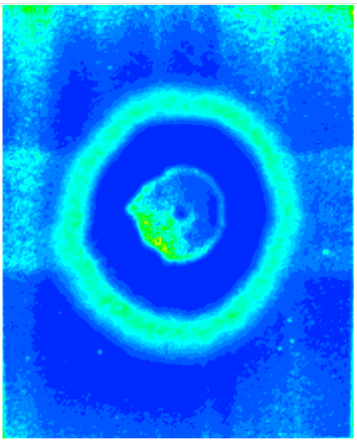

(e) exp., $x / D_{f}=1.5$

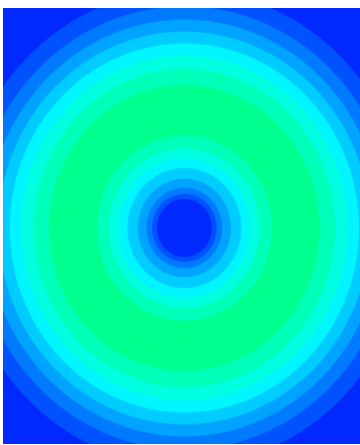

(h) CFD, $x / D_{f}=5.0$

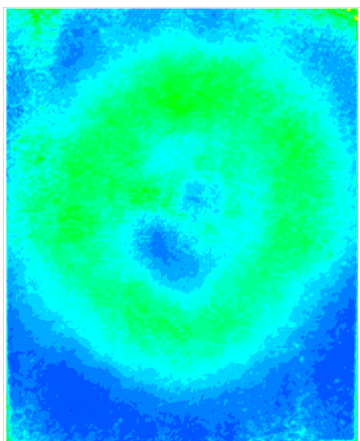

(k) exp., $x / D_{f}=5.0$

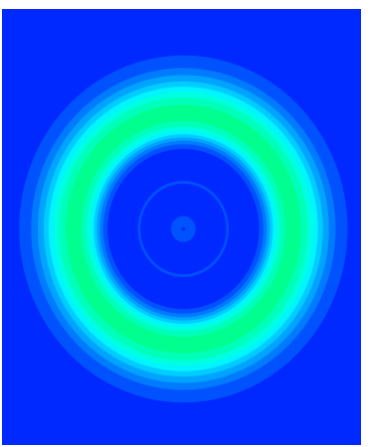

(c) CFD, $x / D_{f}=2.0$

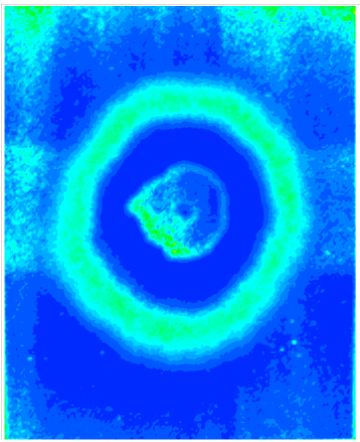

(f) exp., $x / D_{f}=2.0$

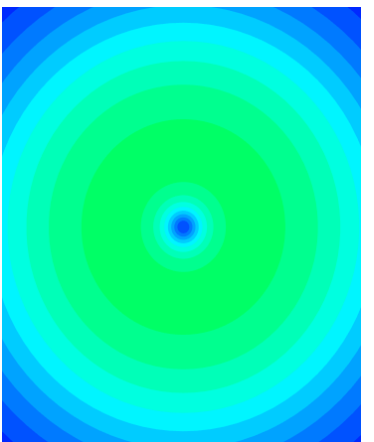

(i) CFD, $x / D_{f}=7.0$

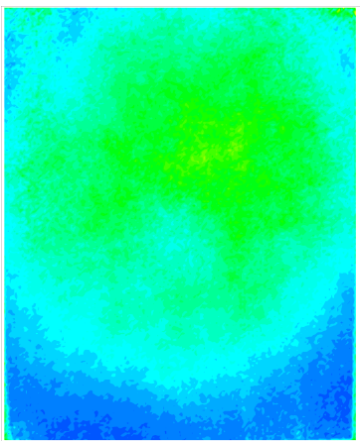

(l) exp., $x / D_{f}=7.0$

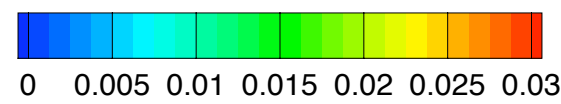

Figure 27. Contours of turbulent kinetic energy, $k / U_{c}^{2}$, for $5 \mathrm{BB}$ baseline nozzle (no wedge), hot conditions 


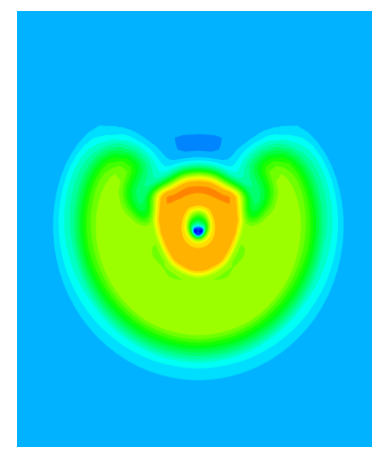

(a) CFD, $x / D_{f}=1.0$

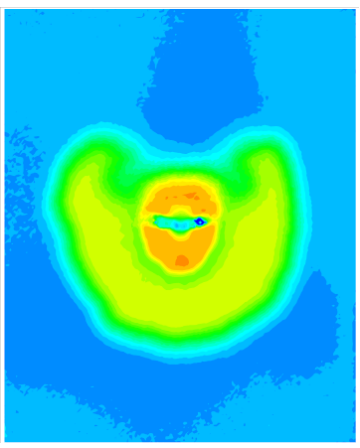

(d) exp., $x / D_{f}=1.0$

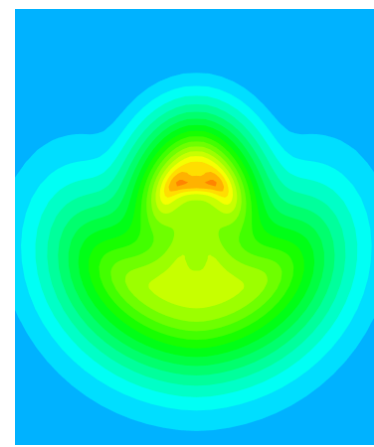

(g) CFD, $x / D_{f}=3.0$

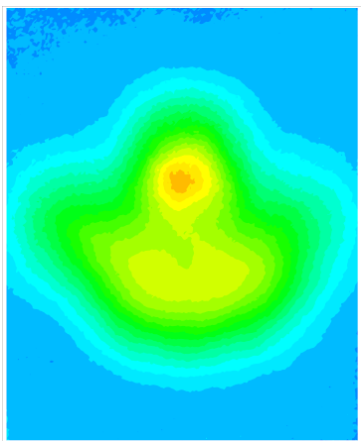

(j) exp., $x / D_{f}=3.0$

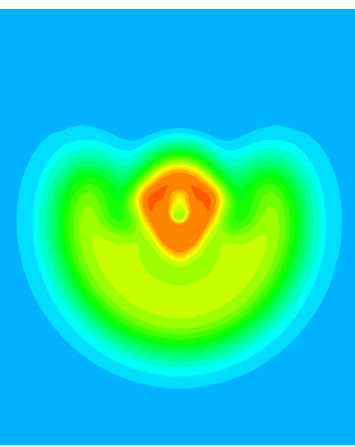

(b) CFD, $x / D_{f}=1.5$

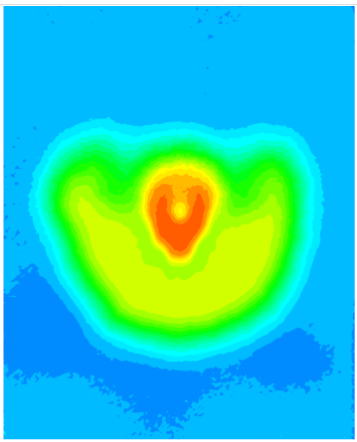

(e) exp., $x / D_{f}=1.5$

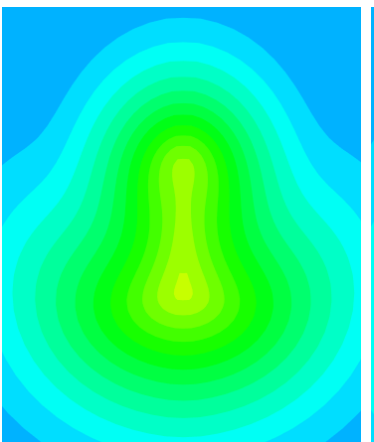

(h) CFD, $x / D_{f}=5.0$

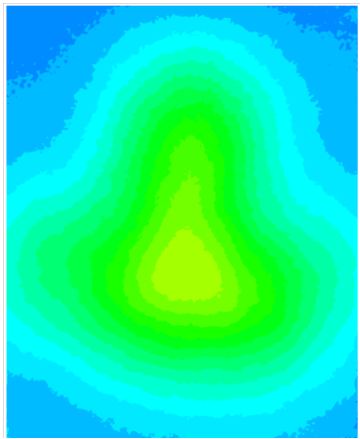

(k) exp., $x / D_{f}=5.0$

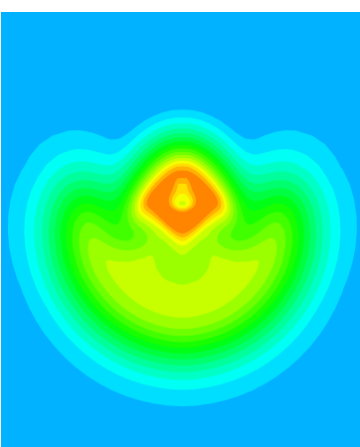

(c) CFD, $x / D_{f}=2.0$

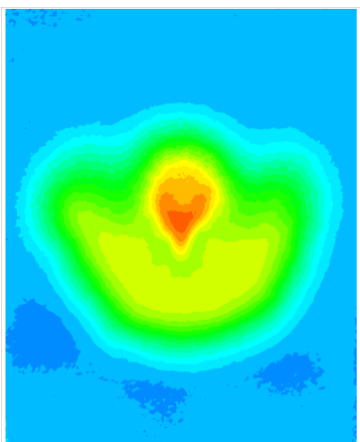

(f) exp., $x / D_{f}=2.0$

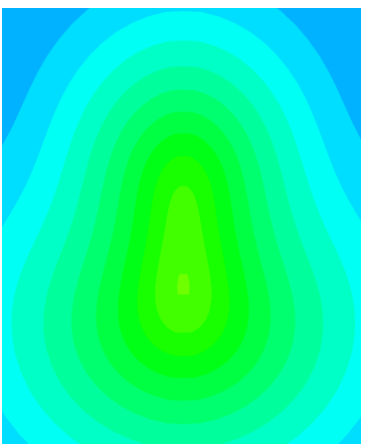

(i) $\mathrm{CFD}, x / D_{f}=7.0$

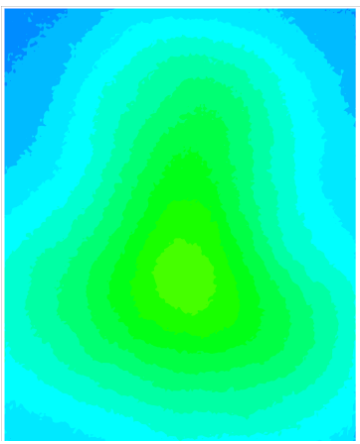

(l) exp., $x / D_{f}=7.0$

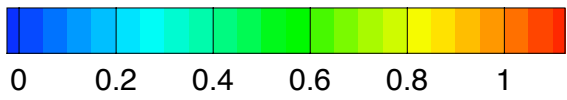

Figure 28. Contours of u-velocity, $u / U_{c}$, for 5BB nozzle with wedge, hot conditions 


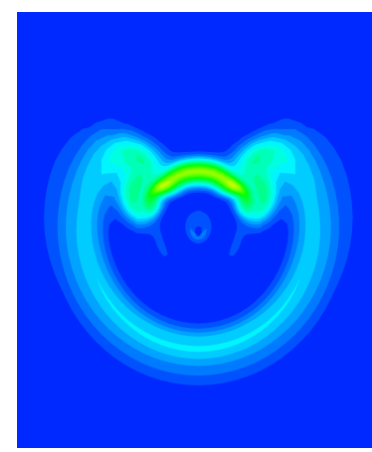

(a) CFD, $x / D_{f}=1.0$

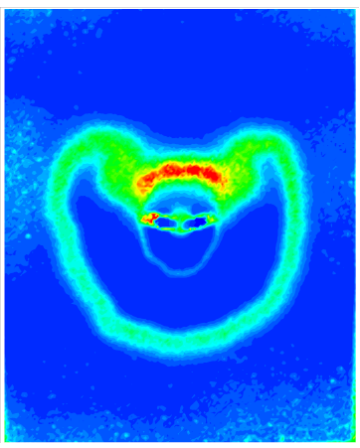

(d) exp., $x / D_{f}=1.0$

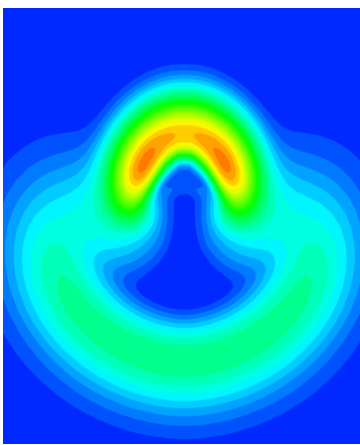

(g) CFD, $x / D_{f}=3.0$

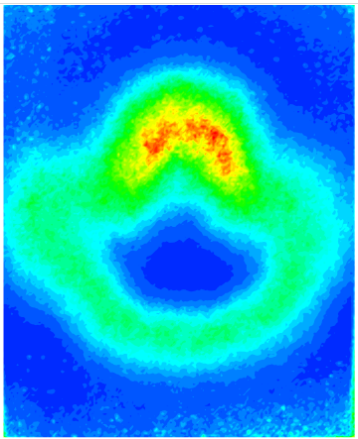

(j) exp., $x / D_{f}=3.0$

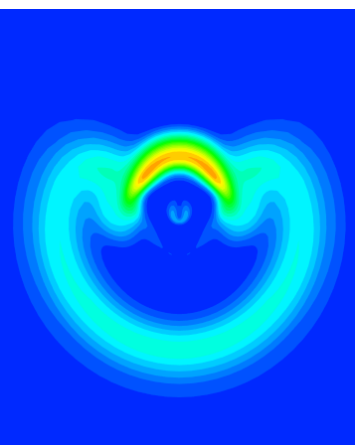

(b) CFD, $x / D_{f}=1.5$

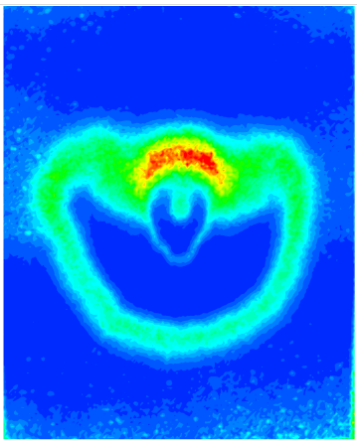

(e) exp., $x / D_{f}=1.5$

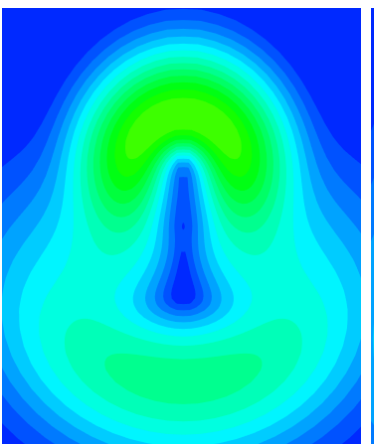

(h) CFD, $x / D_{f}=5.0$

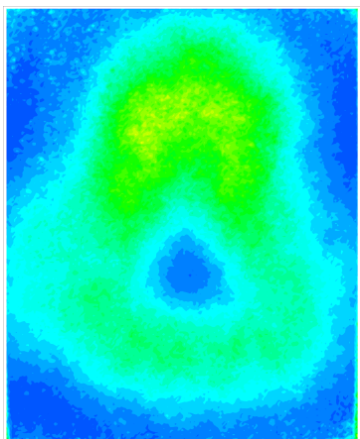

(k) exp., $x / D_{f}=5.0$

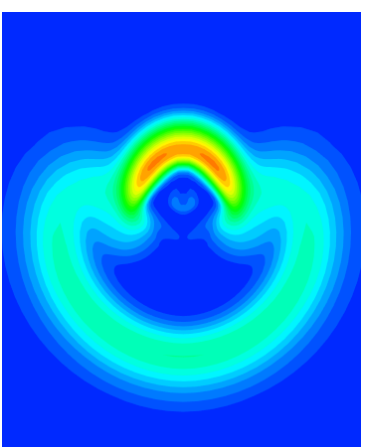

(c) CFD, $x / D_{f}=2.0$

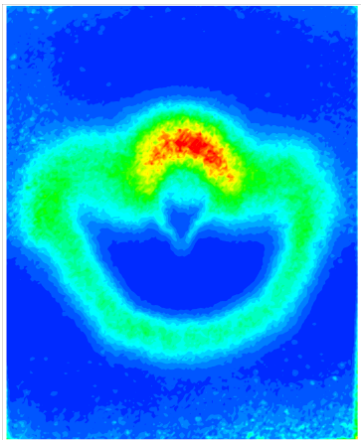

(f) exp., $x / D_{f}=2.0$

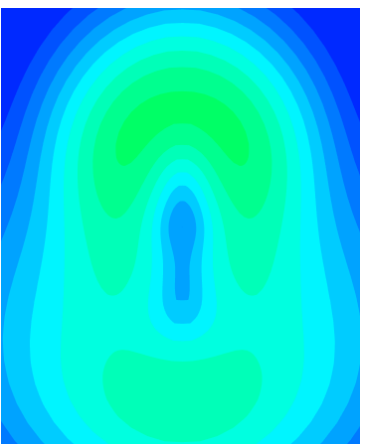

(i) CFD, $x / D_{f}=7.0$

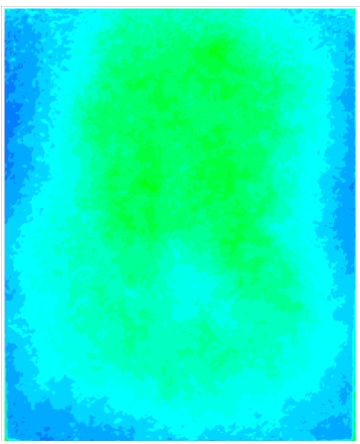

(l) exp., $x / D_{f}=7.0$

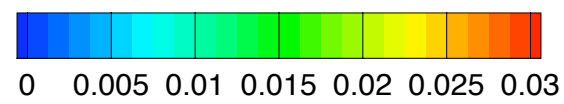

Figure 29. Contours of turbulent kinetic energy, $k / U_{c}^{2}$, for 5BB nozzle with wedge, hot conditions 


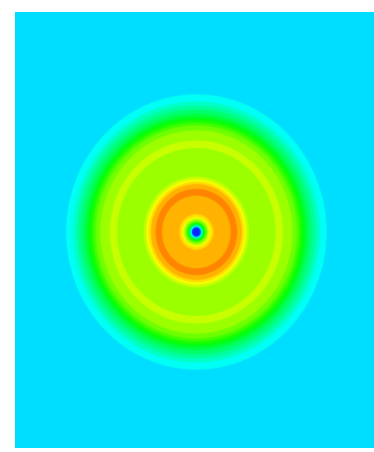

(a) CFD, $x / D_{f}=1.0$

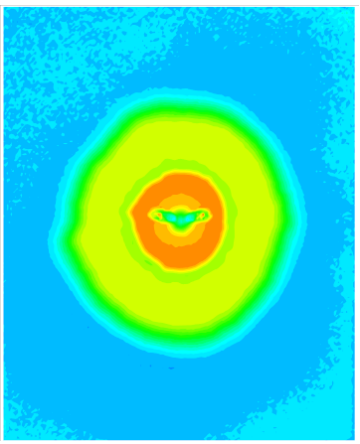

(d) exp., $x / D_{f}=1.0$

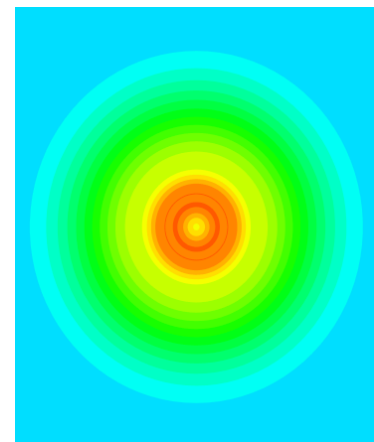

(g) CFD, $x / D_{f}=3.0$

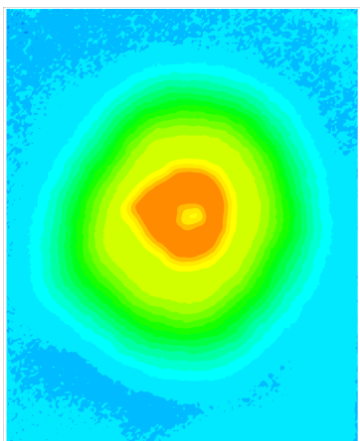

(j) exp., $x / D_{f}=3.0$

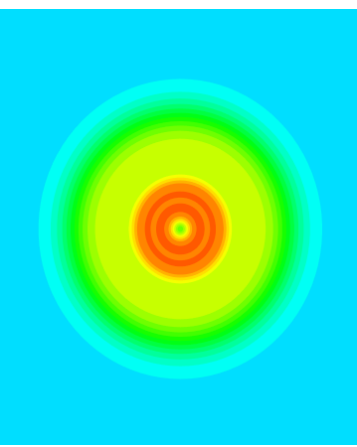

(b) CFD, $x / D_{f}=1.5$

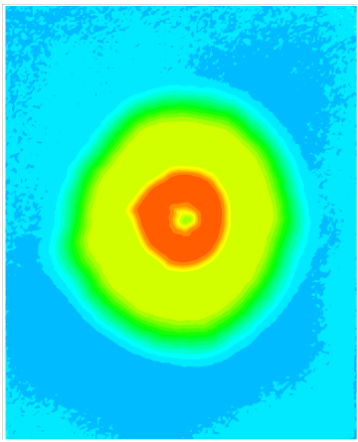

(e) exp., $x / D_{f}=1.5$

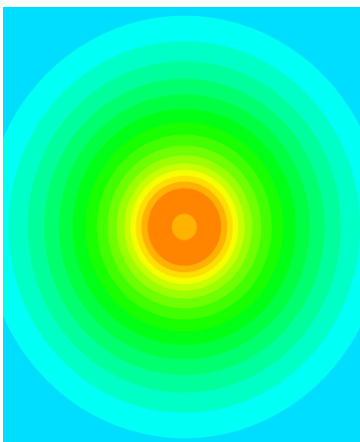

(h) CFD, $x / D_{f}=5.0$

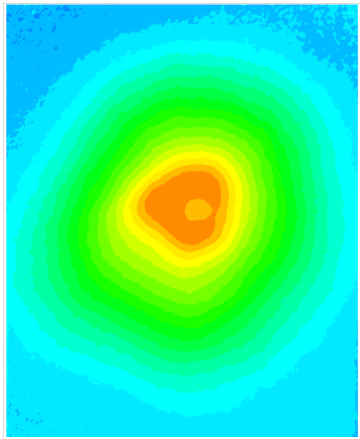

(k) exp., $x / D_{f}=5.0$

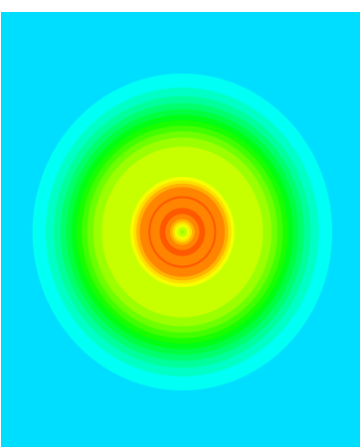

(c) CFD, $x / D_{f}=2.0$

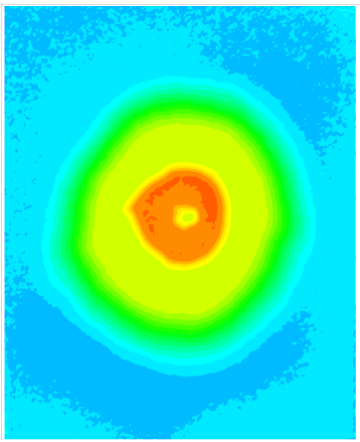

(f) exp., $x / D_{f}=2.0$

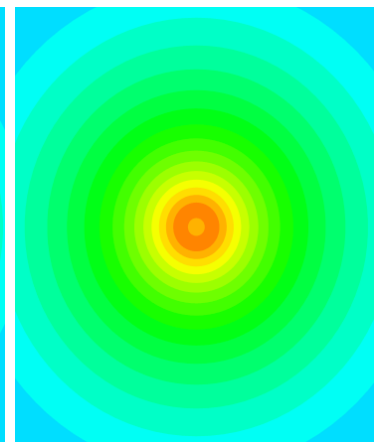

(i) $\mathrm{CFD}, x / D_{f}=7.0$

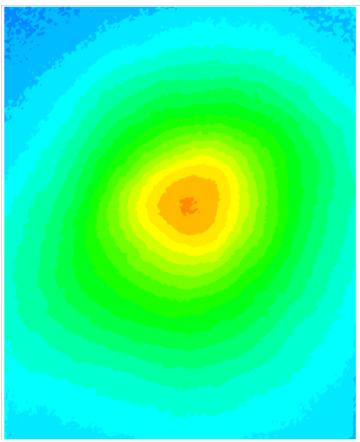

(l) exp., $x / D_{f}=7.0$

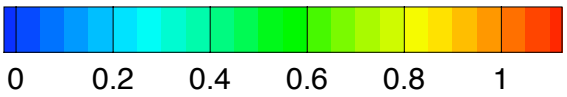

Figure 30. Contours of u-velocity, $u / U_{c}$, for 5BB baseline nozzle (no wedge), cold conditions 


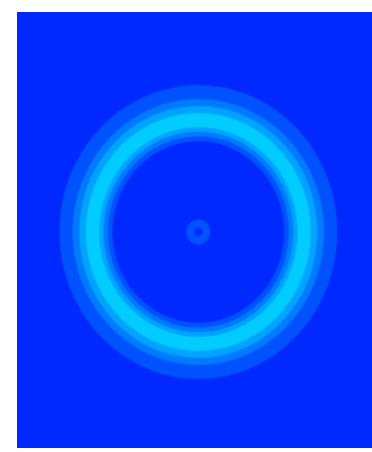

(a) CFD, $x / D_{f}=1.0$

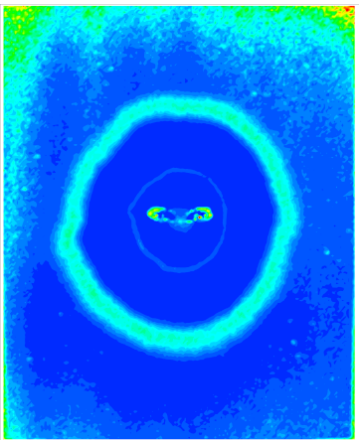

(d) exp., $x / D_{f}=1.0$

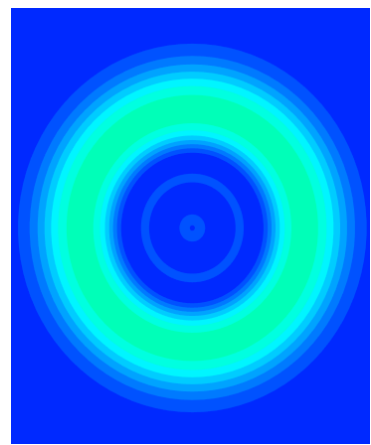

(g) CFD, $x / D_{f}=3.0$

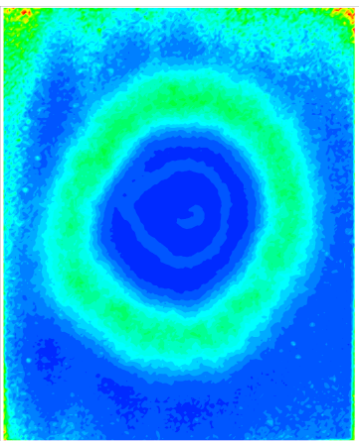

(j) exp., $x / D_{f}=3.0$

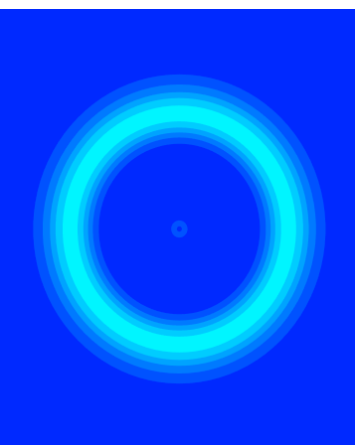

(b) CFD, $x / D_{f}=1.5$

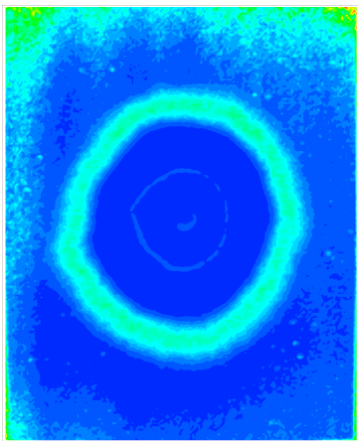

(e) exp., $x / D_{f}=1.5$

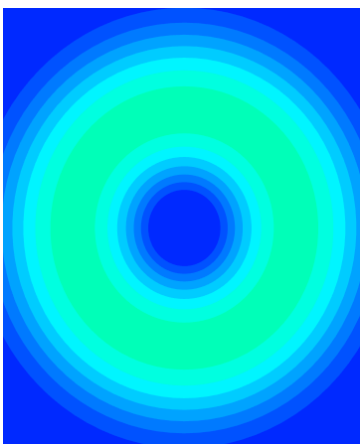

(h) CFD, $x / D_{f}=5.0$

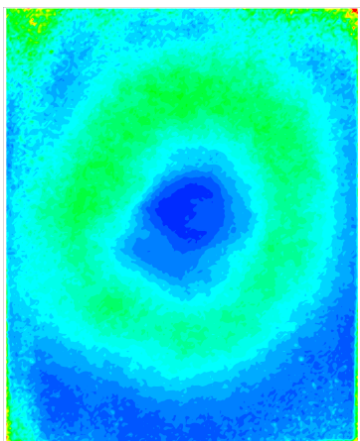

(k) exp., $x / D_{f}=5.0$

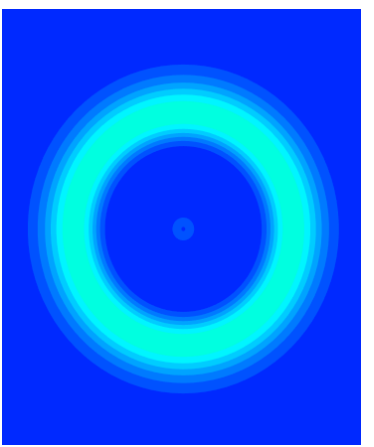

(c) CFD, $x / D_{f}=2.0$

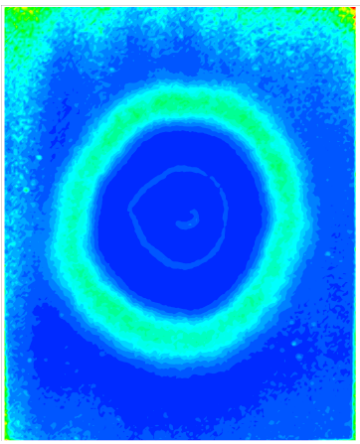

(f) exp., $x / D_{f}=2.0$

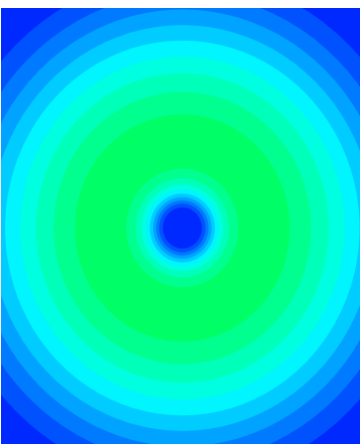

(i) CFD, $x / D_{f}=7.0$

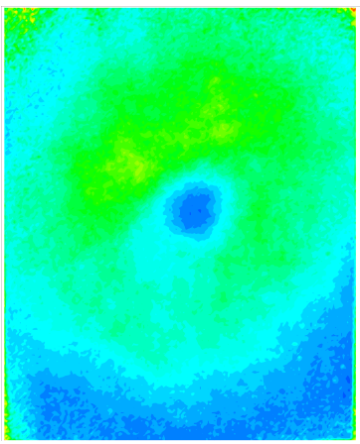

(l) exp., $x / D_{f}=7.0$

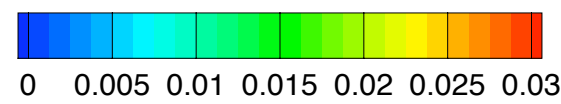

Figure 31. Contours of turbulent kinetic energy, $k / U_{c}^{2}$, for 5BB baseline nozzle (no wedge), cold conditions 


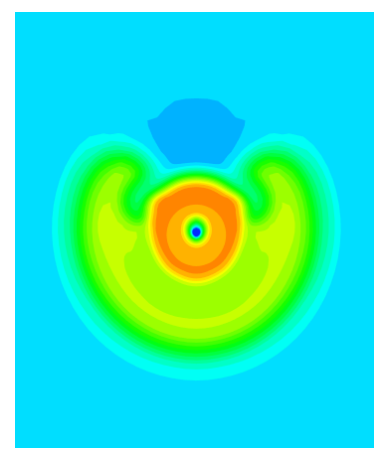

(a) CFD, $x / D_{f}=1.0$

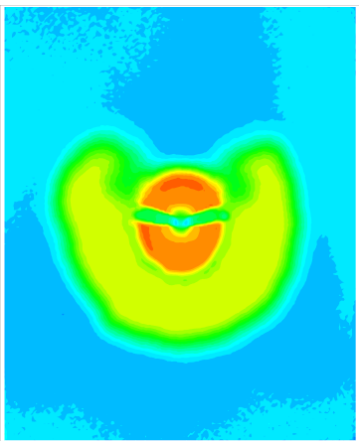

(d) exp., $x / D_{f}=1.0$

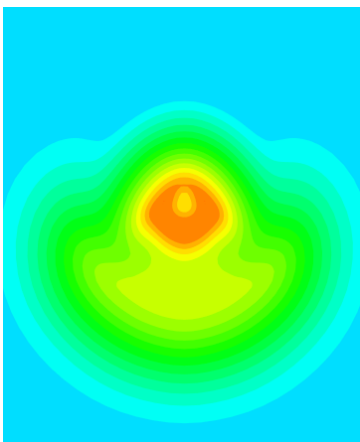

(g) CFD, $x / D_{f}=3.0$

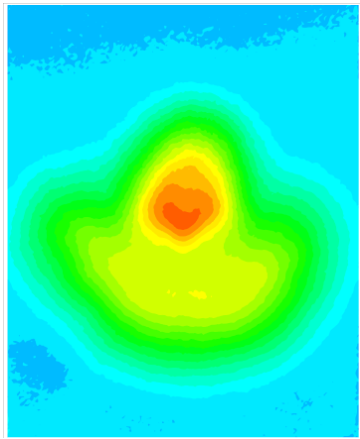

(j) exp., $x / D_{f}=3.0$

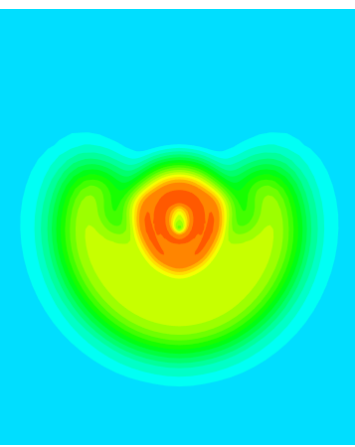

(b) CFD, $x / D_{f}=1.5$

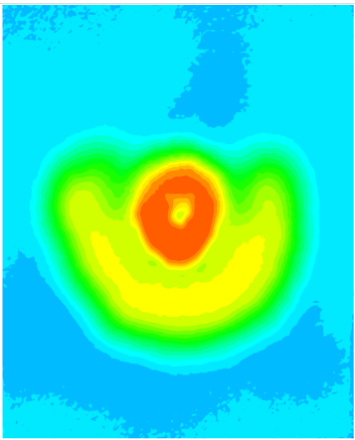

(e) exp., $x / D_{f}=1.5$

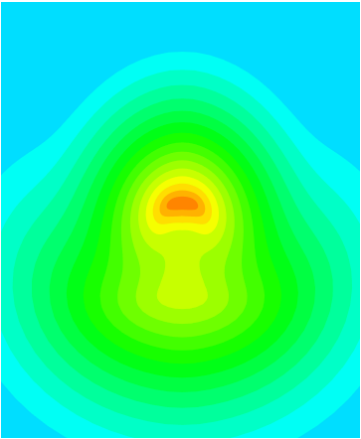

(h) CFD, $x / D_{f}=5.0$

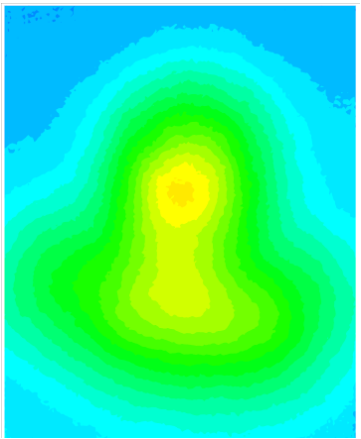

(k) exp., $x / D_{f}=5.0$

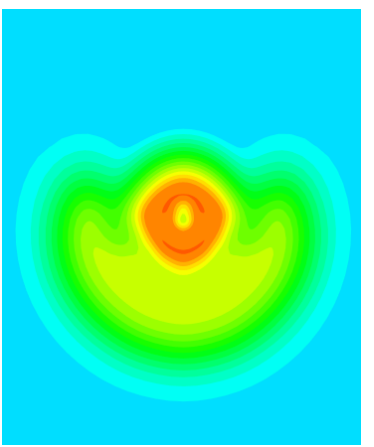

(c) CFD, $x / D_{f}=2.0$

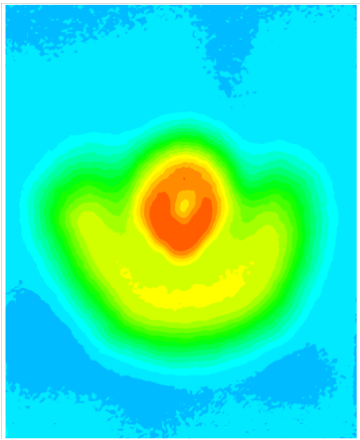

(f) exp., $x / D_{f}=2.0$

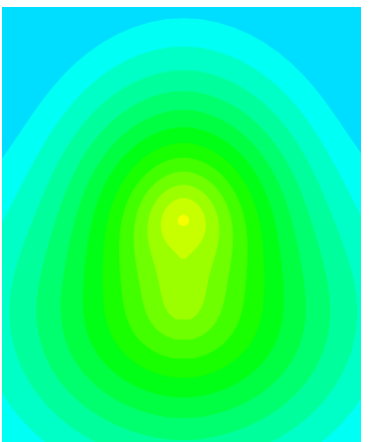

(i) $\mathrm{CFD}, x / D_{f}=7.0$

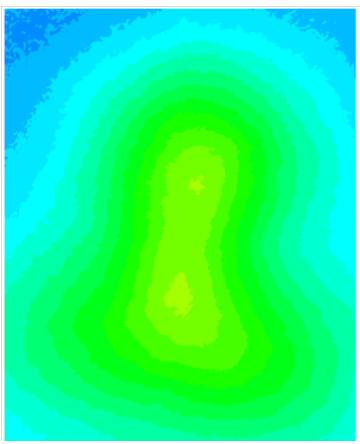

(l) exp., $x / D_{f}=7.0$

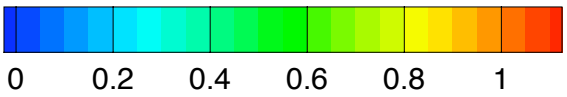

Figure 32. Contours of u-velocity, $u / U_{c}$, for 5BB nozzle with wedge, cold conditions 


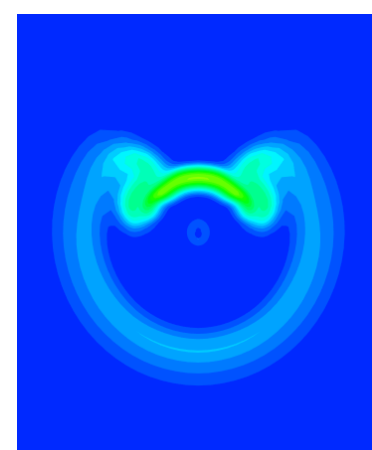

(a) CFD, $x / D_{f}=1.0$

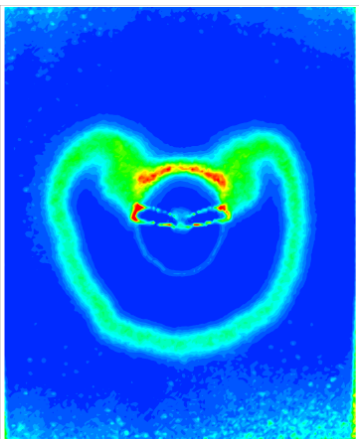

(d) exp., $x / D_{f}=1.0$

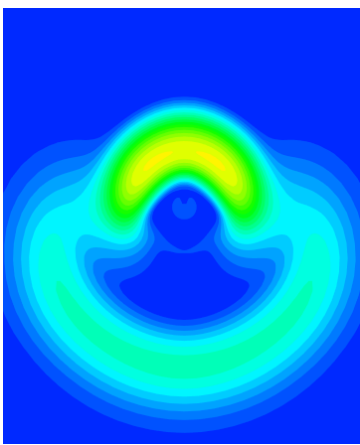

(g) CFD, $x / D_{f}=3.0$

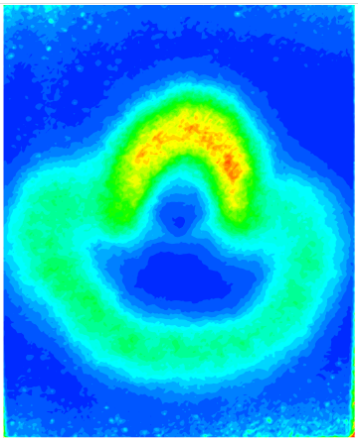

(j) exp., $x / D_{f}=3.0$

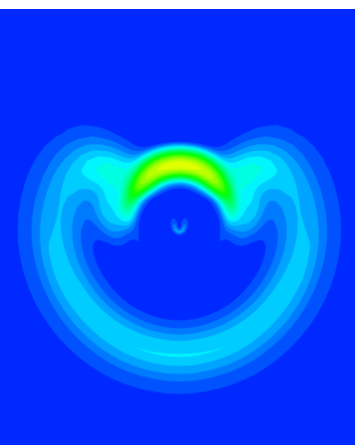

(b) CFD, $x / D_{f}=1.5$

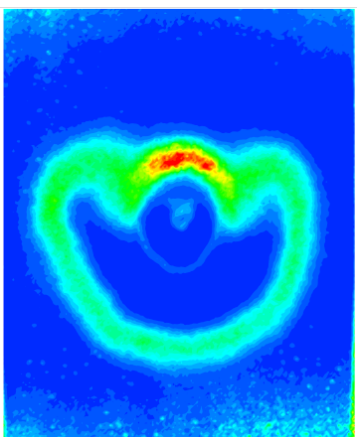

(e) exp., $x / D_{f}=1.5$

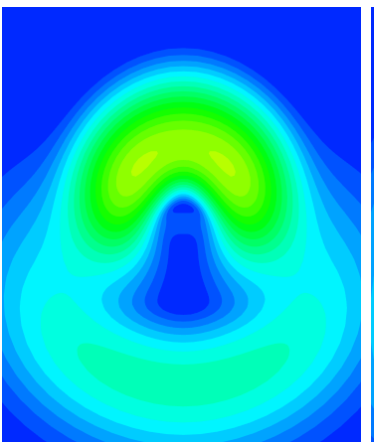

(h) CFD, $x / D_{f}=5.0$

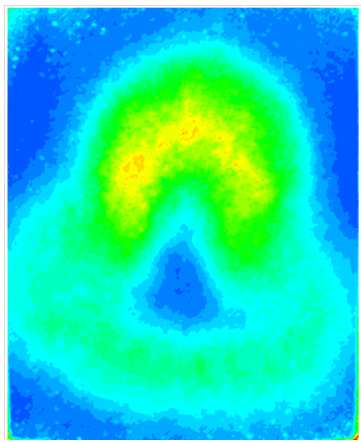

(k) exp., $x / D_{f}=5.0$

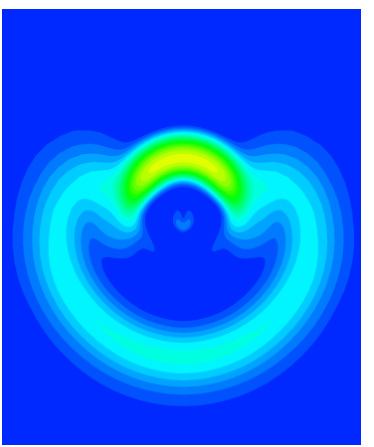

(c) CFD, $x / D_{f}=2.0$

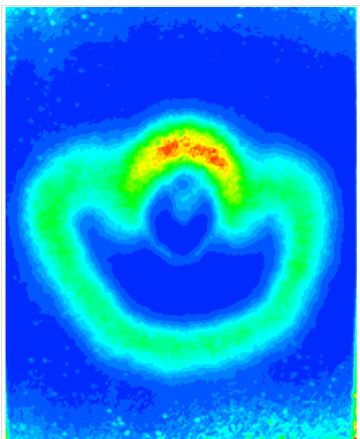

(f) exp., $x / D_{f}=2.0$

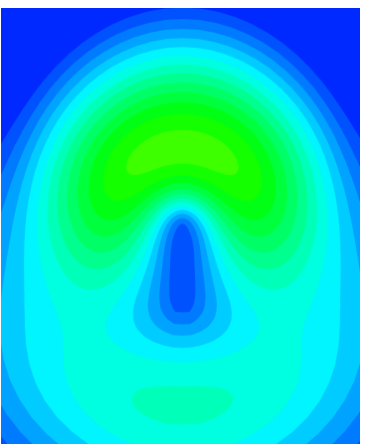

(i) CFD, $x / D_{f}=7.0$

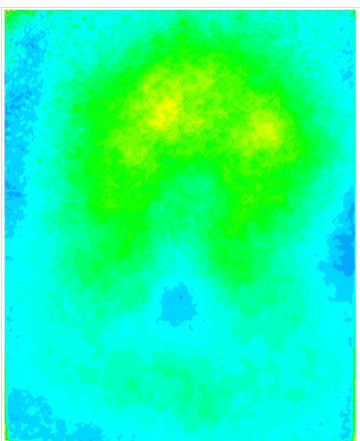

(l) exp., $x / D_{f}=7.0$

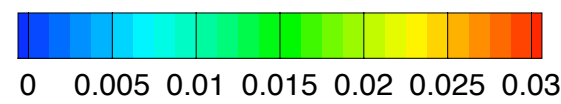

Figure 33. Contours of turbulent kinetic energy, $k / U_{c}^{2}$, for 5BB nozzle with wedge, cold conditions 


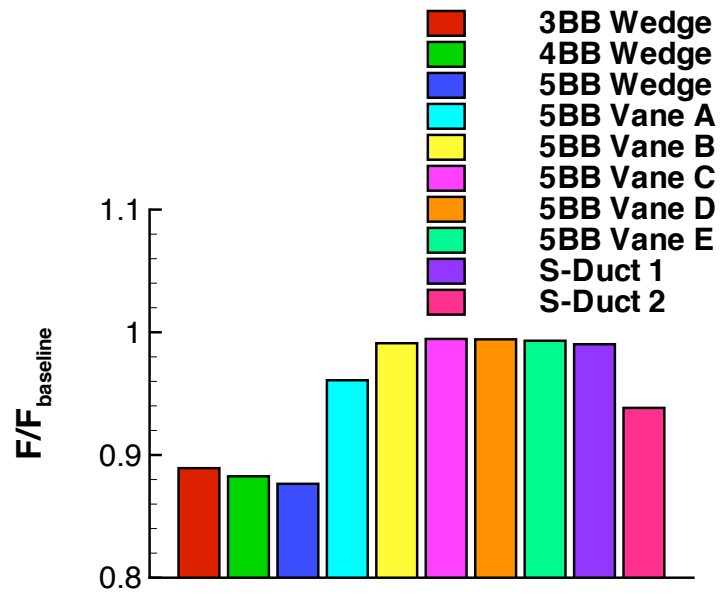

(a) Thrust ratio

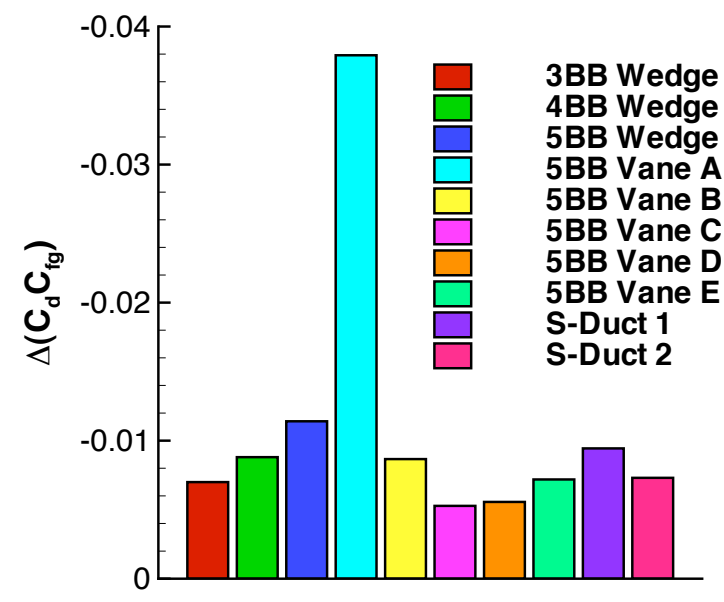

(b) Change in thrust coefficient

Figure 34. Comparison of all Offset Stream Technology Nozzles performance 